\title{
A Born approximation from backscattering data for live loads in Lamé system
}

\author{
Juan Antonio Barceló, Magali Folch-Gabayet, Salvador Pérez-Esteva, \\ Alberto Ruiz and Mari Cruz Vilela
}

\begin{abstract}
We will study the inverse scattering problem for the Lamé equation in elasticity with live loads. We give the definition of a Born approximation of the load from backscattering data. We will see that in $2 \mathrm{D}$, for non-smooth load matrices the main singularities of the matrices are in fact contained in their Born approximations. The singularities are measured in the scale of Sobolev spaces.
\end{abstract}

\section{Introduction and statement of results}

We consider $\mathrm{Q}$ to be a square matrix $\left(q_{i j}(x)\right)$ of order $n$ such that each component $q_{i j}(x)$ is compactly supported and belongs to $L^{r}\left(\mathbb{R}^{n}\right)$, for some $r$ to be determined later. This $\mathrm{Q}$ is assumed to be the matrix of an unknown linear load inside a known homogeneous and isotropic elastic solid. We will try to get information on its coefficients, hence to recover information on parameters inside the unknown perturbation. We obtain partial information on the most singular part of the matrix by defining an appropriate Born approximation based upon the scattering data.

The loaded system is governed by the equation

$$
\Delta^{*} \mathbf{u}(x)+\omega^{2} \mathbf{u}(x)=\mathrm{Q}(x) \mathbf{u}(x), \quad \omega>0, x \in \mathbb{R}^{n}, n \geq 2,
$$

where $\mathbf{u}$, the displacement vector, is a vector-valued function from $\mathbb{R}^{n}$ to $\mathbb{C}^{n}$, and

$$
\Delta^{*} \mathbf{u}(x)=\mu \Delta \mathrm{I} \mathbf{u}(x)+(\lambda+\mu) \nabla \operatorname{div} \mathbf{u}(x)
$$

with $\Delta \mathrm{I}$ denoting the diagonal matrix with the Laplace operator on the diagonal.

The constants $\lambda$ and $\mu$ are known as the Lamé constants. Throughout this paper we will assume that $\mu>0$ and $2 \mu+\lambda>0$, so that the operator $\Delta^{*}$ is strongly

Mathematics Subject Classification (2010): Primary 35J47, 74B05; Secondary 42B37.

Keywords: Inverse scattering problem, Lamé system, Born approximation. 
elliptic. We will denote by $k_{p}$ and $k_{s}$ the speed of propagation of longitudinal waves ( $p$-waves $)$ and transverse waves (s-waves) respectively, which are given by

$$
k_{p}^{2}=\frac{\omega^{2}}{(2 \mu+\lambda)} \quad \text { and } \quad k_{s}^{2}=\frac{\omega^{2}}{\mu} .
$$

We introduce the spectral Navier operator for linear elasticity in a homogeneous and isotropic material,

$$
L \mathbf{u}(x)=\Delta^{*} \mathbf{u}(x)+\omega^{2} \mathbf{u}(x), \quad \omega>0, x \in \mathbb{R}^{n}, n \geq 2,
$$

where $\Delta^{*}$ is given in $(1.2)$.

It is well known that in a domain of $\mathbb{R}^{n}$, any solution $\mathbf{u}$ of the homogeneous spectral Navier equation,

$$
L \mathbf{u}(x)=\Delta^{*} \mathbf{u}(x)+\omega^{2} \mathbf{u}(x)=\mathbf{0},
$$

admits the decomposition

$$
\mathbf{u}=\mathbf{u}_{p}+\mathbf{u}_{s}
$$

where

$$
\mathbf{u}_{p}=-\frac{1}{k_{p}^{2}} \nabla \operatorname{div} \mathbf{u} \quad \text { and } \quad \mathbf{u}_{s}=\mathbf{u}-\mathbf{u}_{p}
$$

are called the compressional part and the shear part of $\mathbf{u}$, and they are solutions of the vectorial homogeneous Helmholtz equations $\Delta \mathrm{I} \mathbf{u}_{p}(x)+k_{p}^{2} \mathbf{u}_{p}(x)=\mathbf{0}$ and $\Delta \mathrm{I} \mathbf{u}_{s}(x)+k_{s}^{2} \mathbf{u}_{s}(x)=\mathbf{0}$, respectively.

A solution $\mathbf{u}$ of $\Delta^{*} \mathbf{u}(x)+\omega^{2} \mathbf{u}(x)=\mathbf{0}$ in an exterior domain satisfies the outgoing Kupradze radiation conditions if $\mathbf{u}_{p}$ and $\mathbf{u}_{s}$ satisfy the corresponding outgoing Sommerfeld radiation conditions; that is,

$$
\begin{array}{ll}
\left(\partial_{r}-i k_{p}\right) \mathbf{u}_{p}=\mathbf{o}\left(r^{-(n-1) / 2}\right), & r=|x| \rightarrow \infty, \\
\left(\partial_{r}-i k_{s}\right) \mathbf{u}_{s}=\mathbf{o}\left(r^{-(n-1) / 2}\right), & r=|x| \rightarrow \infty .
\end{array}
$$

If $\mathbf{u}$ is an entire solution (i.e., a solution in the whole $\mathbb{R}^{n}$ ) of the homogeneous equation given in (1.3) satisfying the outgoing Kupradze radiation conditions, then $\mathbf{u}=\mathbf{0}$ (to see this it is enough to realize that this result is true for the Helmholtz equation, see [5] and [14] for the two-dimensional case and [6] for the three-dimensional case).

As a consequence, for a compactly supported vector-valued function $\mathbf{f}$, if there exists a solution $\mathbf{u}$ of the Navier equation

$$
\Delta^{*} \mathbf{u}(x)+\omega^{2} \mathbf{u}(x)=\mathbf{f}(x), \quad \omega>0, x \in \mathbb{R}^{n}, n \geq 2,
$$

satisfying the outgoing Kupradze radiation conditions (1.6) and (1.7), where $\mathbf{u}_{p}$ and $\mathbf{u}_{s}$ are given by (1.5) off the support of $\mathbf{f}$, then the solution is unique. 
Any solution $\mathbf{u}$ of (1.1) such that

$$
\mathbf{u}(x)=\mathbf{u}_{\mathbf{i}}(x)+\mathbf{v}(x),
$$

where $\mathbf{u}_{\mathbf{i}}$, the incident wave, is a solution of the homogeneous Lamé equation given in (1.3), and $\mathbf{v}$, the scattered solution, satisfies the outgoing Kupradze radiation conditions (1.6) and (1.7), will be called a scattering solution for the Lamé equation.

We will always consider incident plane waves either transverse plane waves (plane s-waves)

$$
\mathbf{u}_{i}^{s}(x)=e^{i k_{s} \varphi \cdot x} \theta
$$

with polarization vector $\varphi \in \mathbb{S}^{n-1}$ orthogonal to the wave direction $\theta \in \mathbb{S}^{n-1}$, or longitudinal plane waves (plane $p$-waves)

$$
\mathbf{u}_{i}^{p}(x)=e^{i k_{p} \theta \cdot x} \theta .
$$

We use the so called limiting absorption principles to construct the scattered solutions $\mathbf{v}^{p}(x ; \omega, \theta)$ and $\mathbf{v}^{s}(x ; \omega, \theta, \varphi)$, corresponding to incident plane $p$-waves and incident plane $s$-waves respectively, for sufficiently high energy (see [2]). We require the high energy condition because we do not assume any symmetry on Q; even more, it could be a complex matrix. Even in the scalar case (Schrödinger equation) for complex potentials (non self-adjoint operators) the uniqueness of the scattered solution cannot be obtained for small frequencies (see [7]).

Since $\mathbf{v}^{p}$ and $\mathbf{v}^{s}$ satisfy (1.3) off the support of Q, we can perform the decomposition given in (1.4) of both solutions. From the asymptotic expressions of these decompositions we define different scattering amplitudes, which will depend on the corresponding parameters, namely $\mathbf{v}_{p, \infty}^{p}(\zeta ; \omega, \theta), \mathbf{v}_{s, \infty}^{p}(\zeta ; \omega, \theta), \mathbf{v}_{p, \infty}^{s}(\zeta ; \omega, \theta, \varphi)$ and, $\mathbf{v}_{s, \infty}^{s}(\zeta ; \omega, \theta, \varphi)$, where $\zeta=x /|x|$. These data are known as $p \rightarrow p, p \rightarrow s$, $s \rightarrow p$ and, $s \rightarrow s$ scattering data, respectively.

One expects to recover the load Q from high energy limits of scattering data. This was the case for the Schrödinger equation, the scalar analogous of (1.1), see [10] and [9]. Nevertheless, in the case of the Lamé system the recovery from high energy limits works for (1.1) only in very special cases, namely either assuming $\mathrm{Q}=q(x) \mathrm{I}$ or $k_{p}=k_{s}$ (see [1]). It is then natural to define a Born approximation of $\mathrm{Q}$ from partial knowledge of the scattering data. In a previous work [1] we studied the case of fixed angle scattering data.

In this work we define a Born approximation $\mathbf{Q}_{b}$ constructed by using the set of backscattering data, which are given by the scattering amplitude with $\theta=-\zeta$, for any wave direction $\theta$ and any polarization vector $\varphi$ in the incident plane waves. We prove that this approximation allows us to reconstruct the main singularities of the matrix $Q(x)$ in the 2D case, where the singularities are measured in Sobolev scales (see (1.10) below).

Theorem 1.1. Let $0 \leq \beta<1$. If $\mathrm{Q}$ is a square matrix function of order 2 in $\mathbb{R}^{2}$ with compact support such that $\mathrm{Q} \in \mathbf{W}^{\beta, 2}\left(\mathbb{R}^{2}\right) \cap \mathbf{L}^{r}\left(\mathbb{R}^{2}\right)$ for some $r>1$, then we have that $\mathrm{Q}-\mathrm{Q}_{b} \in \mathbf{W}^{\alpha, 2}\left(\mathbb{R}^{2}\right)+\boldsymbol{C}^{\infty}\left(\mathbb{R}^{2}\right)$ for all $\alpha<\beta+1 / 2$ if $0<\beta<1 / 2$, and for $\alpha<1$ if $\beta \geq 1 / 2$, where $\mathbf{W}^{\beta, 2}\left(\mathbb{R}^{2}\right)$ denotes the matrix-valued Sobolev spaces. 
In Section 2 we introduce the scattering solutions and define the Born approximation for backscattering data, we recall the limiting absorption principles collected in [2]; we write the actual potential as a Neumann-Born series in term of the resolvent and control the Sobolev norm of the general term in the series. The quadratic term in the Neumann-Born series has to be treated independently. We will write it in terms of operators which have interest on their own, and will be studied in Section 3. The case $k_{p}=k_{s}$ (vectorial Schrödinger equation) can be reduced to the scalar case and it was studied in several works (see [8], [12], [13], and [15]), so we avoid this special case. We include an appendix with several calculations needed in Section 3.

Notation. We denote by $\Lambda^{s}$ the fractional differentiation operator

$$
\Lambda^{s}=(1-\Delta)^{s / 2}=\mathcal{F}^{-1}\langle\xi\rangle^{s} \mathcal{F},
$$

where $\mathcal{F}$ denotes the Fourier transform and $\langle\xi\rangle=\left(1+|\xi|^{2}\right)^{1 / 2}$.

We use the Sobolev spaces

$$
W^{s, p}\left(\mathbb{R}^{n}\right)=\left\{f \in \mathcal{S}^{\prime}\left(\mathbb{R}^{n}\right): \Lambda^{s} f \in L^{p}\left(\mathbb{R}^{n}\right)\right\},
$$

and the weighted Sobolev spaces

$$
W_{\delta}^{s, p}\left(\mathbb{R}^{n}\right)=\left\{f \in \mathcal{S}^{\prime}\left(\mathbb{R}^{n}\right): \Lambda^{s} f \in L_{\delta}^{p}\left(\mathbb{R}^{n}\right)\right\},
$$

where $L_{\delta}^{p}\left(\mathbb{R}^{n}\right)=\left\{f:\langle x\rangle^{\delta} f \in L^{p}\left(\mathbb{R}^{n}\right)\right\}$. We will also consider the homogeneous Sobolev spaces, defined for $s \geq 0$ as

$$
\dot{W}^{s, p}\left(\mathbb{R}^{n}\right)=\left\{f \in \mathcal{S}^{\prime}\left(\mathbb{R}^{n}\right):\left(|\xi|^{s} \widehat{f} \hat{)} \in L^{p}\left(\mathbb{R}^{n}\right)\right\},\right.
$$

and for $s<0$,

$$
\dot{W}^{s, p}\left(\mathbb{R}^{n}\right)=\left(\dot{W}^{-s, p^{\prime}}\left(\mathbb{R}^{n}\right)\right)^{*},
$$

with $1 / p+1 / p^{\prime}=1$. For each Banach function space $B$ appearing in this paper we will consider the vector-valued version $\mathbf{B}$ of functions with values in $\mathbb{C}^{n}$ whose norm is defined by replacing accordingly the complex modulus by the norm in $\mathbb{C}^{n}$.

Let $z \in \mathbb{C}$ with $\Im(z) \neq 0$ if $\Re(z)>0 . R(z)=(\Delta+z)^{-1}$ is the resolvent of the Laplacian. For $k \in \mathbb{R}$, the operator $R\left(k^{2}+i 0\right)$ is defined by

$$
R\left(k^{2}+i 0\right)=\underset{z \rightarrow k^{2}, \Im z-\lim _{\Im}}{\text { weak }}(\Delta+z)^{-1} g .
$$

We will denote by $M f$ the Hardy-Littlewood maximal function of a locally integrable function in $\mathbb{R}^{n}$,

$$
M f(x)=\sup _{r>0} \frac{1}{|B(0, r)|} \int_{B(0, r)}|f(x-y)| d y .
$$

Acknowledgement. We would like to thank J. M. Reyes for enlightening conversations. 


\section{The scattering amplitudes and the Born approximation of $\mathrm{Q}$}

To define the scattering amplitudes for solutions of (1.8) we introduce the Leray's projection operator $\mathrm{I}-\mathcal{R}$, where I denotes the identity matrix of order $n$ and $\mathcal{R}$ is the operator acting on vector fields as a matrix

$$
\mathcal{R}=-\left(R_{i} R_{j}\right)
$$

where $R_{j}$ is the Riesz transform given by

$$
R_{j} g(x)=c_{n} p . v \cdot \int_{\mathbb{R}^{n}} \frac{x_{j}-y_{j}}{|x-y|^{n}} g(y) d y .
$$

Via the Fourier transform we have

$$
(\mathcal{R} \mathbf{f})^{\wedge}(\xi)=\left(\widehat{\mathbf{f}}(\xi) \cdot \frac{\xi}{|\xi|}\right) \frac{\xi}{|\xi|}=\Pi_{\xi /|\xi|} \widehat{\mathbf{f}}(\xi), \quad \xi \in \mathbb{R}^{n}
$$

with $\mathbf{f} \in \mathbf{L}^{2}\left(\mathbb{R}^{n}\right)$ and $\widehat{\mathbf{f}}(\xi)=\left(\widehat{f_{1}}(\xi), \ldots, \widehat{f_{n}}(\xi)\right)$, where $\Pi_{\xi /|\xi|}$ denotes the orthogonal projection on the line defined by $\xi$.

We can generate solutions of equation (1.8) from the following limiting absorption principle.

Theorem 2.1 (Theorem 1.1 in [2]). Let be $1 / p+1 / q=1$ with $2 /(n+1) \leq 1 / p-$ $1 / q \leq 2 / n$ if $n>2$, or $2 / 3 \leq 1 / p-1 / q<1$ if $n=2$. Then the weak limit absorption principle for the operator $\Delta^{*}$ holds in the space $\mathbf{L}^{p}\left(\mathbb{R}^{n}\right)$ for outgoing Kupradze radiation conditions, that is, for $\mathbf{f} \in \mathbf{L}^{p}\left(\mathbb{R}^{n}\right)$ the weak limit

$$
\mathbf{R}\left(\omega^{2}+i 0\right) \mathbf{f}=\underset{z \rightarrow \omega^{2}, \Im z-\lim _{\Im}>0}{ }\left(\Delta^{*}+z\right)^{-1} \mathbf{f}
$$

exists in $\mathbf{L}^{q}\left(\mathbb{R}^{n}\right)$ and is a weak solution of the equation (1.8) satisfying

$$
\left\|\mathbf{R}\left(\omega^{2}+i 0\right) \mathbf{f}\right\|_{\mathbf{L}^{q}\left(\mathbb{R}^{n}\right)} \leq c \omega^{n(1 / p-1 / q)-2}\|\mathbf{f}\|_{\mathbf{L}^{p}\left(\mathbb{R}^{n}\right)} .
$$

Furthermore, if $\mathbf{f} \in \mathbf{L}^{p}\left(\mathbb{R}^{n}\right) \cap \mathbf{C}_{0}^{\infty}\left(\mathbb{R}^{n}\right)$ then $\mathbf{R}\left(\omega^{2}+i 0\right) \mathbf{f}=\mathbf{u}$ is the unique solution of (1.8) satisfying the outgoing Kupradze radiation conditions given in (1.6) and (1.7). We will refer to $\mathbf{u}$ as the outgoing solution.

Moreover, we have the representation (Helmholtz decomposition)

$$
\mathbf{u}=\mathbf{R}\left(\omega^{2}+i 0\right) \mathbf{f}=\frac{1}{2 \mu+\lambda} R\left(k_{p}^{2}+i 0\right) \mathbf{I}(\mathcal{R} \mathbf{f})+\frac{1}{\mu} R\left(k_{s}^{2}+i 0\right) \mathbf{I}(\mathbf{f}-\mathcal{R} \mathbf{f}) .
$$

Given $\mathbf{f} \in \mathbf{C}_{0}^{\infty}\left(\mathbb{R}^{n}\right)$ we can write the outgoing solution $\mathbf{u}=\mathbf{u}_{p}+\mathbf{u}_{s}$ off the support of $\mathbf{f}$. Here $\mathbf{u}$ is defined by (1.8) and $\mathbf{u}_{p}$ and $\mathbf{u}_{s}$ are given by (1.5). One can verify that $\mathbf{u}_{p}$ and $\mathbf{u}_{s}$ satisfy the following vectorial Helmholtz equations (see the proof of Theorem 1.1 in [2]):

$$
\Delta \mathrm{I} \mathbf{u}_{p}+k_{p}^{2} \mathbf{u}_{p}=-\frac{1}{\omega^{2}} \nabla \operatorname{div} \mathbf{f}, \quad \Delta \mathrm{I} \mathbf{u}_{s}+k_{s}^{2} \mathbf{u}_{s}=\frac{1}{\omega^{2}}\left(k_{s}^{2} \mathbf{f}+\nabla \operatorname{div} \mathbf{f}\right) .
$$


Since the right-hand sides of these equations are vector fields in $\mathbf{C}_{0}^{\infty}\left(\mathbb{R}^{n}\right)$ and $\mathbf{u}_{p}$ and $\mathbf{u}_{s}$ satisfy the corresponding outgoing Sommerfeld radiation conditions for these Helmholtz equations, we have the following asymptotic expansions:

$$
\begin{array}{ll}
\mathbf{u}_{p}(x)=c k_{p}^{(n-3) / 2} \frac{e^{i k_{p}|x|}}{|x|^{(n-1) / 2}} \mathbf{u}_{p, \infty}\left(k_{p}, x /|x|\right)+\mathbf{o}\left(|x|^{-(n-1) / 2}\right), & |x| \rightarrow \infty, \\
\mathbf{u}_{s}(x)=c k_{s}^{(n-3) / 2} \frac{e^{i k_{s}|x|}}{|x|^{(n-1) / 2}} \mathbf{u}_{s, \infty}\left(k_{s}, x /|x|\right)+\mathbf{o}\left(|x|^{-(n-1) / 2}\right), & |x| \rightarrow \infty,
\end{array}
$$

where $\mathbf{u}_{p, \infty}$ and $\mathbf{u}_{s, \infty}$ are known as the longitudinal and transverse scattering amplitudes of $\mathbf{u}$ respectively. These can be written as

$$
\begin{aligned}
\mathbf{u}_{p, \infty}\left(k_{p}, x /|x|\right) & =-\frac{1}{\omega^{2}}(\nabla \operatorname{div} \mathbf{f}) \uparrow\left(k_{p} x /|x|\right), \\
\mathbf{u}_{s, \infty}\left(k_{s}, x /|x|\right) & =\frac{1}{\omega^{2}}\left(\left(\nabla \operatorname{div}+k_{s}^{2} \mathrm{I}\right) \mathbf{f}\right) \uparrow\left(k_{s} x /|x|\right) \\
& =\frac{1}{\omega^{2}}((\nabla \operatorname{div}-\Delta \mathrm{I}) \mathbf{f}) \uparrow\left(k_{s} x /|x|\right) .
\end{aligned}
$$

Definition 2.2. Let $\mathbf{u}$ be an outgoing solution of the Lamé equation (1.8). For $\mathbf{f} \in \mathbf{L}^{p}\left(\mathbb{R}^{n}\right)$ with $1 \leq p<2(n+1) /(n+3)$, we define the longitudinal scattering amplitude of $\mathbf{u}$ as

$$
\mathbf{u}_{p, \infty}\left(k_{p}, x /|x|\right)=-\frac{1}{2 \mu+\lambda}(\mathcal{R} \mathbf{f})^{\wedge}\left(k_{p} x /|x|\right),
$$

and the transverse scattering amplitude of $\mathbf{u}$ as

$$
\mathbf{u}_{s, \infty}\left(k_{s}, x /|x|\right)=\frac{1}{\mu}((\mathrm{I}-\mathcal{R}) \mathbf{f}) \uparrow\left(k_{s} x /|x|\right) .
$$

Remark 2.3. Observe that for $\mathbf{f} \in \mathbf{C}_{0}^{\infty}\left(\mathbb{R}^{n}\right)$, since $\Delta \mathcal{R}=\nabla$ div, the definitions given in (2.4) and (2.5) are equal to the expressions given in (2.2) and (2.3), respectively.

Remark 2.4. Taking into account that we can rewrite $(\mathcal{R} \mathbf{f})^{\curlywedge}(\xi)=\Pi_{\xi /|\xi|}(\widehat{\mathbf{f}}(\xi))$, we have that

$$
\begin{aligned}
& \mathbf{u}_{p, \infty}\left(k_{p}, x /|x|\right)=-\frac{1}{2 \mu+\lambda} \Pi_{x /|x|} \widehat{\mathbf{f}}\left(k_{p} x /|x|\right), \\
& \mathbf{u}_{s, \infty}\left(k_{s}, x /|x|\right)=\frac{1}{\mu}\left(\mathrm{I}-\Pi_{x /|x|}\right) \widehat{\mathbf{f}}\left(k_{s} x /|x|\right) .
\end{aligned}
$$

These expressions show the necessity of considering $\mathbf{f} \in \mathbf{L}^{p}\left(\mathbb{R}^{n}\right)$ with $1 \leq p<$ $2(n+1) /(n+3)$ in Definition 2.2, because the restriction of the Fourier transform $\widehat{\mathbf{f}}$ to a sphere is well defined just for $p$ in this range of values (see [16]).

Remark 2.5. In the particular case $\mu+\lambda=0$, the Navier equation given in (1.8) is actually the vectorial Helmholtz equation $\Delta \mathrm{I} \mathbf{u}+k_{s}^{2} \mathbf{u}=\mathbf{f} / \mu$. In this case $k_{p}=k_{s}$, and

$$
\mathbf{u}_{s, \infty}\left(k_{s}, x /|x|\right)-\mathbf{u}_{p, \infty}\left(k_{s}, x /|x|\right)=\frac{1}{\mu} \widehat{\mathbf{f}}\left(k_{s} x /|x|\right) .
$$


Let us observe that the scattered solution $\mathbf{v}=\mathbf{v}\left(\cdot ; \omega, \mathbf{u}_{i}\right)$, see $(1.9)$, satisfies the following equation:

$$
\left(\Delta^{*}+\omega^{2} \mathrm{I}\right) \mathbf{v}=\mathrm{Q} \mathbf{u}_{\mathbf{i}}+\mathrm{Q} \mathbf{v}
$$

If we apply the outgoing resolvent $\mathbf{R}\left(\omega^{2}+i 0\right)$ we get an integral equation

$$
\left[\mathrm{I}-\mathbf{R}\left(\omega^{2}+i 0\right) \mathrm{Q}\right] \mathbf{v}=\mathbf{R}\left(\omega^{2}+i 0\right) \mathbf{Q} \mathbf{u}_{\mathbf{i}}
$$

This can be solved by a Neumann series in the case of high energy,

$$
\mathbf{v}=\sum_{j=0}^{\infty}\left(\mathbf{R}\left(\omega^{2}+i 0\right) \mathrm{Q}\right)^{j+1}\left(\mathbf{u}_{\mathbf{i}}\right)
$$

We have the following existence result.

Proposition 2.6 ([1], Proposition 3.1). Let $\mathrm{Q}(x)=\left(q_{i j}(x)\right)$ be a square matrix of order $n$ such that each component $q_{i j}(x)$ is compactly supported and belongs to $L^{r}\left(\mathbb{R}^{n}\right)$ with $r>n / 2$ and $n \geq 2$, and set $t=\min \{(n+1) / 2, r\}$. Then, there exist $\omega_{0}=\omega_{0}\left(\operatorname{supp} \mathrm{Q},\|\mathrm{Q}\|_{L^{r}}\right)$ and a constant $c=c(\operatorname{supp} \mathrm{Q})$ such that, for $\omega>\omega_{0}$, there is a unique solution $\mathbf{v}$ of the equation $(2.9)$ in $\mathbf{L}^{q}\left(\mathbb{R}^{n}\right)$, where $1 / 2-1 / q=1 / 2 t$. Besides,

$$
\|\mathbf{v}\|_{\mathbf{L}^{q}} \leq c \omega^{n / t-2}\|\mathrm{Q}\|_{L^{r}}
$$

where $\|\mathrm{Q}\|_{L^{r}}=\max _{1 \leq i, j \leq n}\left\|q_{i, j}\right\|_{L^{r}}$.

Under the conditions of Proposition 2.6, we have that $\mathrm{Q} \mathbf{u}_{i}+\mathrm{Q} \mathbf{v} \in \mathbf{L}^{p}\left(\mathbb{R}^{n}\right)$ for some $p$ in the interval $[1,2(n+1) /(n+3))$, and therefore, we can define longitudinal and transverse scattering amplitudes for $\mathbf{v}$, the solution of the Navier equation given in (2.8).

If we consider an incident plain $p$-wave $\mathbf{u}_{i}^{p}(x)=e^{i k_{p} \theta \cdot x} \theta$ with $\theta \in \mathbb{S}^{n-1}$, the Helmholtz decomposition given in (2.1) and formulae (2.6) and (2.7) gives us a longitudinal scattering amplitude $(p \rightarrow p$ scattering data)

$$
\mathbf{v}_{p, \infty}^{p}(\zeta ; \omega, \theta)=\frac{-1}{2 \mu+\lambda} \Pi_{\zeta} \widehat{\mathrm{Q}}\left(k_{p}(\zeta-\theta)\right) \theta-\frac{1}{2 \mu+\lambda} \Pi_{\zeta}\left(\mathrm{Q} \mathbf{v}^{p}(\cdot ; \omega, \theta)\right) \uparrow\left(k_{p} \zeta\right)
$$

and a transverse scattering amplitude $(p \rightarrow s$ scattering data)

$$
\mathbf{v}_{s, \infty}^{p}(\zeta ; \omega, \theta)=\frac{1}{\mu}\left(\mathrm{I}-\Pi_{\zeta}\right) \widehat{\mathrm{Q}}\left(k_{s} \zeta-k_{p} \theta\right) \theta+\frac{1}{\mu}\left(\mathrm{I}-\Pi_{\zeta}\right)\left(\mathrm{Q} \mathbf{v}^{p}(\cdot ; \omega, \theta)\right) \widehat{\imath}\left(k_{s} \zeta\right)
$$

where $x /|x|=\zeta$ and $\mathbf{v}^{p}(\cdot ; \omega, \theta)$ is the scattered solution which satisfies the integral equation

$$
\left(\mathrm{I}-\mathbf{R}\left(\omega^{2}+i 0\right) \mathrm{Q}\right) \mathbf{v}^{p}=\mathbf{R}\left(\omega^{2}+i 0\right) \mathrm{Q}\left(e^{i \frac{\omega}{\sqrt{2 \mu+\lambda}} \theta \cdot(\cdot)} \theta\right) .
$$


If we consider as the incident wave a plane $s$-wave, $\mathbf{u}_{\mathbf{i}}(x)=e^{i k_{s} \theta \cdot x} \varphi$, where $\theta$ and $\varphi$ are unitary orthogonal vectors, we have as longitudinal scattering amplitude $(s \rightarrow p$ scattering data)

$$
\begin{aligned}
\mathbf{v}_{p, \infty}^{s}(\zeta ; \omega, \theta, \varphi)= & \frac{-1}{2 \mu+\lambda} \Pi_{\zeta} \widehat{\mathrm{Q}}\left(k_{p} \zeta-k_{s} \theta\right) \varphi \\
& -\frac{1}{2 \mu+\lambda} \Pi_{\zeta}\left(\mathrm{Qv}^{s}(\cdot ; \omega, \theta, \varphi)\right)^{\curlywedge}\left(k_{p} \zeta\right)
\end{aligned}
$$

and as transverse scattering amplitude $(s \rightarrow s$ scattering data)

$$
\begin{aligned}
\mathbf{v}_{s, \infty}^{s}(\zeta ; \omega, \theta, \varphi)= & \frac{1}{\mu}\left(\mathrm{I}-\Pi_{\zeta}\right) \widehat{\mathrm{Q}}\left(k_{s}(\zeta-\theta)\right) \varphi \\
& +\frac{1}{\mu}\left(\mathrm{I}-\Pi_{\zeta}\right)\left(\mathrm{Qv}^{s}(\cdot ; \omega, \theta, \varphi)\right) \widehat{(}\left(k_{s} \zeta\right),
\end{aligned}
$$

where now $\mathbf{v}^{s}(\cdot ; \omega, \theta, \varphi)$ is the scattered solution satisfying the integral equation

$$
\left(\mathrm{I}-\mathbf{R}\left(\omega^{2}+i 0\right) \mathrm{Q}\right) \mathbf{v}^{s}=\mathbf{R}\left(\omega^{2}+i 0\right) \mathrm{Q}\left(e^{i \frac{\omega}{\sqrt{\mu}} \theta \cdot(\cdot)} \varphi\right) .
$$

Observe that the identities given above hold whenever $\omega>\omega_{0}$, since this condition is needed to guarantee the existence of the scattered solutions (see Proposition 2.6).

\subsection{Born approximation for backscattering data}

The realization $\zeta=-\theta$ produces polar coordinates in the Fourier transform variable with radius proportional to the frequency $\omega$. It is convenient to show every appearance of $\omega$ in $k_{p}$ and $k_{s}$.

Let us take $\zeta=-\theta$, and assume that $\omega$ is sufficiently large $(\omega>B, B$ to be chosen). Changing $\omega$ to $\sqrt{2 \mu+\lambda} \omega$ in $(2.11)$ and $\omega$ to $2\left(\frac{1}{\sqrt{\mu}}+\frac{1}{\sqrt{2 \mu+\lambda}}\right)^{-1} \omega$ in (2.12), we have, for $K=\sqrt{2 \mu+\lambda} / \sqrt{\mu}$,

$$
\widehat{\mathrm{Q}}(-2 \omega \theta) \theta=\mathbf{v}_{\infty}^{p}(\theta, \omega)+\mathbf{h}^{p}(\theta, \omega),
$$

where the term $\mathbf{v}_{\infty}^{p}(\theta, \omega)$, depending only on backscattering data is given by

$$
\mathbf{v}_{\infty}^{p}(\theta, \omega)=-(2 \mu+\lambda) \mathbf{v}_{p, \infty}^{p}(-\theta ; \omega \sqrt{2 \mu+\lambda}, \theta)+\mu \mathbf{v}_{s, \infty}^{p}\left(-\theta ; \frac{2 \sqrt{\mu}}{1+K^{-1}} \omega, \theta\right)
$$

and the error is given by

$$
\begin{aligned}
\mathbf{h}^{p}(\theta, \omega)= & \Pi_{-\theta}\left[\left(\mathrm{Qv}^{p}\left(\cdot ; \lambda_{p p} \omega, \theta\right)\right) \wedge(-\omega \theta)\right] \\
& +\left(\mathrm{I}-\Pi_{-\theta}\right)\left[\left(\mathrm{Qv}^{p}\left(\cdot ; \lambda_{p s} \omega, \theta\right)\right) \wedge\left(-\mu_{p s} \omega\right)\right],
\end{aligned}
$$

where $\mathbf{v}^{p}(\cdot ; \omega, \theta)$ is the scattered solution of the integral equation (2.13), and the parameters are defined as

$$
\lambda_{p p}=\sqrt{2 \mu+\lambda}, \quad \lambda_{p s}=\frac{2 \sqrt{\mu}}{1+K^{-1}}, \quad \mu_{p p}=1, \quad \mu_{p s}=\frac{2}{1+K^{-1}} .
$$


Similarly, from (2.14) and (2.15) we get

$$
\widehat{\mathrm{Q}}(-2 \omega \theta) \varphi=\mathbf{v}_{\infty}^{s}(\theta ; \omega, \varphi)-\mathbf{h}^{s}(\theta ; \omega, \varphi),
$$

where

$$
\mathbf{v}_{\infty}^{s}(\theta ; \omega, \varphi)=-(2 \mu+\lambda) \mathbf{v}_{p, \infty}^{s}\left(-\theta ; \frac{2 \omega \sqrt{\mu}}{1+K^{-1}}, \theta, \varphi\right)+\mu \mathbf{v}_{s, \infty}^{s}(-\theta ; \omega \sqrt{\mu}, \theta, \varphi)
$$

and the error is given by

$$
\begin{aligned}
\mathbf{h}^{s}(\theta ; \omega, \varphi)= & \Pi_{-\theta}\left[\left(\mathrm{Q}^{s}\left(\cdot ; \lambda_{s p} \omega, \theta, \varphi\right)\right)\left(\left(-\mu_{s p} \omega \theta\right)\right]\right. \\
& +\left(\mathrm{I}-\Pi_{-\theta}\right)\left[\left(\mathrm{Q} \mathbf{v}^{s}\left(\cdot ; \lambda_{s s} \omega, \theta, \varphi\right)\right) \hat{(}(-\omega \theta)\right],
\end{aligned}
$$

with $\mathbf{v}^{s}(\cdot ; \omega, \theta, \varphi)$ being the solution of the integral equation (2.16) and the parameters are

$$
\lambda_{s p}=\frac{2 \sqrt{2 \mu+\lambda}}{1+K}, \quad \lambda_{s s}=\sqrt{\mu}, \quad \mu_{s p}=\frac{2}{1+K}, \quad \mu_{s s}=1 .
$$

For $\theta \in \mathbb{S}^{n-1}$ fixed, we consider $n-1$ unit vectors $\left\{\varphi_{2}, \ldots, \varphi_{n}\right\}$ such that $\varphi_{\ell}=\varphi_{\ell}(\theta)$ is measurable in $\mathbb{S}^{n-1}$ for $\ell=2, \ldots, n$, and such that the set $\left\{\theta, \varphi_{2}, \ldots, \varphi_{n}\right\}$ is an orthonormal base of $\mathbb{R}^{n}$.

We have

$$
\widehat{\mathrm{Q}}(-2 \omega \theta) e_{j}=\left(\theta \cdot e_{j}\right) \widehat{\mathrm{Q}}(-2 \omega \theta) \theta+\sum_{\ell=2}^{n}\left(\varphi_{\ell} \cdot e_{j}\right) \widehat{\mathrm{Q}}(-2 \omega \theta) \varphi_{\ell} .
$$

Using (2.17) and (2.19) we obtain

$$
\widehat{\mathrm{Q}}(-2 \omega \theta) e_{j}=\widehat{\mathrm{Q}}_{b}(\omega, \theta) e_{j}-\widehat{E}_{b}(\omega, \theta) e_{j},
$$

with

$$
\widehat{\mathrm{Q}}_{b}(\omega, \theta) e_{j}=\left(\theta \cdot e_{j}\right) \mathbf{v}_{\infty}^{p}(\theta, \omega)+\sum_{\ell=2}^{n}\left(\varphi_{\ell} \cdot e_{j}\right) \mathbf{v}_{\infty}^{s}\left(\theta ; \omega, \varphi_{\ell}\right)
$$

and

$$
\widehat{E}_{b}(\omega, \theta) e_{j}=\left(\theta \cdot e_{j}\right) \mathbf{h}^{p}(\theta, \omega)+\sum_{\ell=2}^{n}\left(\varphi_{\ell} \cdot e_{j}\right) \mathbf{h}^{s}\left(\theta ; \omega, \varphi_{\ell}\right) .
$$

The above expressions are defined for $\omega$ sufficiently large. Let $B$ be a real positive number large enough, which will be fixed later on, such that $(2.21)$ is defined for $\omega>B / 2$. We insert here a cutoff function $\chi$ supported on $\omega>B$ and identically 1 for $\omega>2 B$. Then we write

$$
\widehat{\mathrm{Q}}(-2 \omega \theta) e_{j} \chi(-2 \omega \theta)=\widehat{\mathrm{Q}}_{b}(\omega, \theta) e_{j} \chi(-2 \omega \theta)-\widehat{E}_{b}(\omega, \theta) e_{j} \chi(-2 \omega \theta),
$$

Taking the inverse Fourier transform in polar coordinates we have

$$
\mathrm{Q}(x) e_{j}=\mathrm{Q}_{b}(x) e_{j}-E_{b}(x) e_{j}+\Phi(x) e_{j},
$$


where the matrix functions are defined as

$$
\begin{aligned}
\Phi(x) e_{j} & =2^{n} \int_{0}^{2 B} \int_{\mathbb{S}^{n-1}} e^{-i x \cdot 2 \omega \theta} \widehat{\mathrm{Q}}(\omega, \theta)(1-\chi(-2 \omega \theta)) e_{j} d \sigma(\theta) \omega^{n-1} d \omega, \\
\mathrm{Q}_{b}(x) e_{j} & =2^{n} \int_{0}^{\infty} \int_{\mathbb{S}^{n-1}} e^{-i x \cdot 2 \omega \theta} \widehat{\mathrm{Q}}_{b}(\omega, \theta) \chi(-2 \omega \theta) e_{j} d \sigma(\theta) \omega^{n-1} d \omega, \\
E_{b}(x) e_{j} & =2^{n} \int_{0}^{\infty} \int_{\mathbb{S}^{n-1}} e^{-i x \cdot 2 \omega \theta} \widehat{E}_{b}(\omega, \theta) \chi(-2 \omega \theta) e_{j} d \sigma(\theta) \omega^{n-1} d \omega
\end{aligned}
$$

Definition 2.7. We define the Born approximation from backscattering of the potential Q as a matrix $Q_{b}$ such that, for each element of the canonical base of $\mathbb{R}^{n}$, $e_{j}=(0, \ldots, 1, \ldots, 0), \mathrm{Q}_{b} e_{j}$ is the vector-valued function from $\mathbb{R}^{n}$ to $\mathbb{C}^{n}$ given in $(2.25)$ and $(2.22)$.

\subsection{Recovery of singularities}

In this subsection we see under which conditions the main singularities of the potential $\mathrm{Q}$ are recovered from its Born backscattering approximation. That is, we want to prove that $\mathrm{Q}_{b}$ contains, in the scale of Sobolev spaces, all the singularities of $\mathrm{Q}$.

From (2.24),

$$
\mathrm{Q}(x) e_{j}=\mathrm{Q}_{b}(x) e_{j}-E_{b}(x) e_{j},
$$

modulo a $\mathcal{C}^{\infty}\left(\mathbb{R}^{n}\right)$ matrix function.

From (2.18) and (2.20) we can write

$$
\widehat{E}_{b}(\omega, \theta) e_{j}=\widehat{E}_{p}^{p} e_{j}+\widehat{E}_{s}^{p} e_{j}+\sum_{\ell=2}^{n}\left(\widehat{E}_{p, \ell}^{s} e_{j}+\widehat{E}_{s, \ell}^{s} e_{j}\right)
$$

where

$$
\begin{aligned}
\widehat{E}_{p}^{p}(\omega, \theta) e_{j} & =\left(\theta \cdot e_{j}\right) \Pi_{-\theta}\left[\left(\mathrm{Q} \mathbf{v}^{p}\left(\cdot ; \lambda_{p p} \omega, \theta\right)\right)^{\wedge}\left(-\mu_{p p} \omega \theta\right)\right], \\
\widehat{E}_{s}^{p}(\omega, \theta) e_{j} & =\left(\theta \cdot e_{j}\right)\left(\mathrm{I}-\Pi_{-\theta}\right)\left[\left(\mathrm{Q} \mathbf{v}^{p}\left(\cdot ; \lambda_{p s} \omega, \theta\right)\right)^{\wedge}\left(-\mu_{p s} \omega\right)\right], \\
\widehat{E}_{p, \ell}^{s}(\omega, \theta) e_{j} & =\left(\varphi_{\ell} \cdot e_{j}\right) \Pi_{-\theta}\left[\left(\mathrm{Q} \mathbf{v}^{s}\left(\cdot ; \lambda_{s p} \omega, \theta, \varphi_{\ell}\right)\right)^{\curlywedge}\left(-\mu_{s p} \omega \theta\right)\right],
\end{aligned}
$$

and

$$
\widehat{E}_{s, \ell}^{s}(\omega, \theta) e_{j}=\left(\varphi_{\ell} \cdot e_{j}\right)\left(\mathrm{I}-\Pi_{-\theta}\right)\left[\left(\mathrm{Q} \mathbf{v}^{s}\left(\cdot ; \lambda_{s s} \omega, \theta, \varphi_{\ell}\right)\right)\left(-\mu_{s s} \omega \theta\right)\right] .
$$

To estimate the $\mathbf{W}^{\alpha, 2}\left(\mathbb{R}^{n}\right)$ norm of the functions defined by $(2.26)-(2.29)$, we ignore the products $\varphi_{\ell} \cdot e_{j}$ and the orthogonal projections, so we are reduced to estimate the integrals

$$
\int_{B}^{\infty} \int_{\mathbb{S}^{n-1}}\left|\left(\mathrm{Qv}^{p}\left(\cdot ; \lambda_{p a} \omega, \theta\right)\right)^{\wedge}\left(-\mu_{p a} \omega \theta\right)\right|^{2} d \sigma(\theta)\left(1+(2 \omega)^{2}\right)^{\alpha} \omega^{n-1} d \omega,
$$

for $a=s, p$. From (2.13) we take the Neumann-Born series solutions and we obtain, for $a=p, s$,

$$
\left(\mathrm{Qv}^{p}\left(\cdot ; \lambda_{p a} \omega, \theta\right)\right) \uparrow\left(-\mu_{p a} \omega \theta\right)=\sum_{k=1}^{\infty} \widehat{\mathcal{Q}}_{a, k+1}^{1}(\omega, \theta),
$$


with

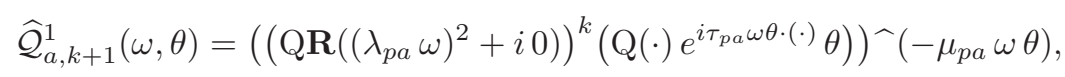

where $\tau_{p p}=1$ and $\tau_{p s}=2 /(1+K)$.

In a similar way, we have for $\ell=2, \ldots, n$ and $a=p, s$,

$$
\left(\mathrm{Qv}^{s}\left(\cdot ; \lambda_{s a} \omega, \theta, \varphi_{\ell}\right)\right)^{\wedge}\left(-\mu_{s a} \omega \theta\right)=\sum_{k=1}^{\infty} \widehat{\mathcal{Q}}_{a, k+1}^{\ell}(\omega, \theta)
$$

with

$$
\widehat{\mathcal{Q}}_{a, k+1}^{\ell}(\omega, \theta)=\left(\left(\mathrm{QR}\left(\left(\lambda_{s a} \omega\right)^{2}+i 0\right)\right)^{k}\left(\mathrm{Q}(\cdot) e^{i \tau_{s a} \omega \theta \cdot(\cdot)} \varphi_{\ell}\right)\right)\left(-\mu_{s a} \omega \theta\right),
$$

where $\tau_{s p}=2 K /(1+K)$ and $\tau_{s s}=1$.

Therefore we can reduce the analysis to estimate for $k=2, \ldots, \ell=1, \ldots, n$ and $a=s, p$

$$
\left\|\mathcal{Q}_{a, k}^{\ell}\right\|_{\mathbf{W}^{\alpha, 2}}^{2} \sim \int_{B}^{\infty} \int_{\mathbb{S}^{n-1}}\left|\widehat{\mathcal{Q}_{a, k}^{\ell}}(\omega, \theta)\right|^{2} d \sigma(\theta)\left(1+(2 \omega)^{2}\right)^{\alpha} \omega^{n-1} d \omega .
$$

We start by giving estimates of the above integrals for $k \geq 3$ as was done in [15] and [13], using estimates for the resolvent in the Sobolev spaces $\mathbf{W}_{\delta}^{\beta, p}\left(\mathbb{R}^{n}\right)$.

Proposition 2.8. Assume that $\mathrm{Q} \in \mathbf{W}^{\beta, 2}\left(\mathbb{R}^{n}\right)$ is compactly supported and $\beta<n / 2$, $n=2,3$. Then there exists a constant $C=C(\alpha, \beta)$ such that

$$
\left\|\mathcal{Q}_{a, k}^{\ell}\right\|_{\mathbf{W}^{\alpha, p}}^{2} \leq C^{k} \int_{B}^{\infty} \omega^{2 \alpha+n-1+2 \gamma_{k}} d \omega\|\mathrm{Q}\|_{\mathbf{L}^{2}}\|\mathrm{Q}\|_{\mathbf{W}^{\beta, 2}}^{k-1}
$$

where

$$
\gamma_{k}=-k+1+\frac{(n-1)(k-3)}{2}\left(\frac{1}{2}-\frac{\beta}{n}\right)+\frac{n-1}{2} \max \left\{0, \frac{1}{2}-\frac{2 \beta}{n}\right\},
$$

$a=p, s$ and $\ell=1, \ldots, n$.

The proof follows the lines of Proposition 4 in [13] by recalling the following estimates for the resolvent given in Lemmas 2.9 and 2.10.

Writing $\theta_{1}=\theta$ and $\theta_{\ell}=\varphi_{\ell}(\theta)$ for $\ell=1, \ldots, n$, we introduce, for $a, b \in\{p, s\}$,

$$
\mathbf{R}_{\ell, \theta}^{a b}(\omega)(\Phi)(x)=e^{-i \tau_{a b} \omega \theta \cdot x} \mathbf{R}\left(\left(\lambda_{a b} \omega\right)^{2}+i 0\right)\left(e^{i \tau_{a b} \omega \theta \cdot(\cdot)} \Phi(\cdot) \theta_{\ell}\right)(x),
$$

where $\Phi(x)$ a square matrix of order $n$.

Lemma 2.9. Let $s \geq 0$, and let $r$ and $t$ be such that $0 \leq 1 / t-1 / 2 \leq 1 /(n+1)$ and $0 \leq 1 / 2-1 / r \leq 1 /(n+1)$. There exist $\delta$, $\delta^{\prime}$ and $C$ (independent of $\omega$ ) such that

$$
\left\|\mathbf{R}_{\ell, \theta}^{a b}(\omega)(\Phi)\right\|_{\boldsymbol{W}_{-\delta}^{s, r}} \leq C \omega^{-1+(n-1) / 2(1 / t-1 / r)}\|\Phi\|_{\boldsymbol{W}_{\delta^{\prime}}^{s, t}} .
$$


Lemma 2.10. Let $s_{1}, s_{2}, s_{3} \geq 0, s_{3} \leq s_{1}, s_{3} \leq s_{2}$, and let $r$, $t$ and $p$ be such that $t<\min \{p, r\}$ and

$$
s_{1}+s_{2}-s_{3} \geq n\left(\frac{1}{p}+\frac{1}{r}-\frac{1}{t}\right) \geq 0 .
$$

Then

$$
\|\Phi \mathbf{f}\|_{\mathbf{W}^{s_{3}, t}} \leq C\|\Phi\|_{\mathbf{W}^{s_{1}, p}}\|\mathbf{f}\|_{\mathbf{W}^{s_{2}, r}} .
$$

Moreover, if $\Phi$ is compactly supported and $\delta, \delta^{\prime} \in \mathbb{R}$, then

$$
\|\Phi \mathbf{f}\|_{\mathbf{W}_{\delta}^{s_{3}, t}} \leq C\left(\operatorname{supp} f, \delta, \delta^{\prime}\right)\|\Phi\|_{\mathbf{W}^{s_{1}, p}}\|\mathbf{f}\|_{\mathbf{W}_{\delta^{\prime}}^{s_{2}, r}} .
$$

The proof of Lemma 2.9 is similar to that of Lemma 5.2 in [1]. Lemma 2.10 can be found in [3] and [11].

Proof of Proposition 2.8. Since

$$
\mathbf{R}\left(\left(\lambda_{a b} \omega\right)^{2}+i 0\right)\left(\Phi(\cdot) \varphi_{\ell}\right)(x)=\mathbf{R} \mathrm{I}\left(\left(\lambda_{a b} \omega\right)^{2}+i 0\right)(\Phi(\cdot))(x) \varphi_{\ell},
$$

where

$$
\left.\mathbf{R I}\left(\lambda_{a b} \omega\right)^{2}+i 0\right)(\Phi(\cdot))(x)
$$

denotes the matrix whose $j$-column is the vector $\mathbf{R}\left(\left(\lambda_{a b} \omega\right)^{2}+i 0\right)\left(\Phi_{1 j}, \ldots, \Phi_{n j}\right)^{t}$, we can write

$$
\begin{aligned}
& \leq C \int_{B}^{\infty} \int_{\mathbb{S}^{n-1}}\left|\int_{\mathbb{R}^{n}} e^{-i\left(\mu_{a b}+\tau_{a b}\right) \omega \theta \cdot y}\left(\mathrm{Q} \mathbf{R}_{\ell, \theta}^{a b}(\omega)\right)^{k-1}\left(\mathrm{Q} \varphi_{\ell}\right)(y) d y\right|^{2} d \sigma(\theta) \omega^{2 \alpha+n-1} d \omega \\
& \leq C \int_{B}^{\infty} \int_{\mathbb{S}^{n-1}}\left\|\left(\mathbf{R}_{\ell, \theta}^{a b}(\omega)\right)^{k-1}(\mathrm{Q})(\cdot)\right\|_{\mathbf{L}^{1}}^{2} d \sigma(\theta) \omega^{2 \alpha+n-1} d \omega .
\end{aligned}
$$

Now we follow the argument of Proposition 4 in [13].

We insert a cut off function $\phi$ which is one to one on the support of $\mathrm{Q}$ and write, for some values of $\delta$,

$$
\begin{aligned}
\|\left(\mathrm{Q}_{\ell, \theta}^{a b}(\omega)\right)^{k-1} & \left(\mathrm{Q} \varphi_{\ell}\right)(\cdot) \|_{\mathbf{L}^{1}} \\
& \leq\|\mathrm{Q}\|_{\mathbf{L}^{2}}\left\|\phi \mathbf{R}_{\ell, \theta}^{a b}(\omega)\left(\mathbf{R}_{\ell, \theta}^{a b}(\omega)\right)^{k-2}\left(\mathrm{Q} \varphi_{\ell}\right)(\cdot)\right\|_{\mathbf{L}^{2}} \\
& \leq C \omega^{-1+(n-1) / 2(1 / t-1 / 2)}\|\mathrm{Q}\|_{\mathbf{L}^{2}}\left\|\left(\mathrm{Q} \mathbf{R}_{\ell, \theta}^{a b}(\omega)\right)^{k-2}\left(\mathrm{Q} \varphi_{\ell}\right)(\cdot)\right\|_{\mathbf{L}_{-\delta}^{t}}
\end{aligned}
$$

where we used Lemma 2.9. We write $\mathbf{G}=\mathbf{R}_{\ell, \theta}^{a b}(\omega)$ and we use an iteration of both Lemmas 2.9 and 2.10 following the diagram:

$$
\begin{aligned}
& \mathbf{W}_{\delta^{\prime}}^{\beta, t_{k-2}} \stackrel{\mathbf{R}_{\theta}}{\longrightarrow} \mathbf{W}_{-\delta}^{\beta, r_{k-2}} \stackrel{Q \cdot}{\longrightarrow} \mathbf{W}_{\delta}^{\beta, t_{k-3}} \cdots \longrightarrow \mathbf{W}_{\delta}^{\beta, t_{2}} \stackrel{\mathbf{R}_{\theta}}{\longrightarrow} \mathbf{W}_{\delta}^{\beta, r_{2}} \stackrel{Q}{\longrightarrow} \quad \mathbf{L}_{-\delta}^{t} \\
& Q \theta \rightsquigarrow \mathbf{G} Q \theta \rightsquigarrow Q \mathbf{G} Q \theta \cdots \rightsquigarrow(Q \mathbf{G})^{k-3} Q \theta \rightsquigarrow \mathbf{G}(\cdot) \rightsquigarrow(Q \mathbf{G})^{k-2} Q \theta,
\end{aligned}
$$


where $r_{\ell}$ and $t_{\ell}$ are to be chosen to satisfy, for $\ell=2, \ldots, k-1$,

$$
\begin{aligned}
& 0 \leq \frac{1}{t_{\ell}}-\frac{1}{2} \leq \frac{1}{n+1} \quad \text { and } \quad 0 \leq \frac{1}{2}-\frac{1}{r_{\ell}} \leq \frac{1}{n+1} \\
& t_{\ell}<\min \left\{2, r_{\ell+1}\right\} \\
& 0 \leq \frac{1}{2}+\frac{1}{r_{\ell+1}}-\frac{1}{t_{\ell}}=\frac{\beta}{n}, \\
& 0 \leq \frac{1}{2}+\frac{1}{r_{2}}-\frac{1}{t}=\frac{2 \beta}{n} \quad \text { and } \quad 0 \leq \frac{1}{t}-\frac{1}{2} \leq \frac{1}{n+1} \text {. }
\end{aligned}
$$

We obtain

$$
\left\|\left(\mathrm{Q} \mathbf{R}_{\ell, \theta}^{a b}(\omega)(\omega)\right)^{k-1}\left(\mathrm{Q} \theta_{a, \ell}\right)(\cdot)\right\|_{\mathbf{L}^{1}} \leq C \omega^{\gamma_{k}}\|\mathrm{Q}\|_{\mathbf{W}^{\beta, 2}}^{k-1},
$$

where

$$
\gamma_{k}=-(k-1)+\frac{n-1}{2}\left(\sum_{l=2}^{k-2}\left(\frac{1}{t_{l}}-\frac{1}{r_{l}}\right)+\frac{1}{t_{1}}-\frac{1}{2}\right) .
$$

For $\epsilon>0$ small, we can choose $\left\{r_{i}, t_{i}\right\}$ such that $1 / t_{l}-1 / r_{l+1}=1 / 2-\beta / n+\epsilon$, $2 \leq l \leq k-2$ and $1 / t-1 / r_{2}=\max \{\epsilon, 1 / 2-2 \beta / n\}$. Taking $\epsilon \rightarrow 0^{+}$and putting together all the above in (2.33), we obtain the desired (2.30) and (2.31).

A consequence of Proposition 2.8 is:

Corollary 2.11. Let $n=2$ and assume that $\mathrm{Q} \in \mathbf{W}^{\beta, 2}\left(\mathbb{R}^{2}\right)$ is compactly supported and $\beta<1$. Then there exists a constant $C=C(\alpha, \beta)$ such that, for any $k \geq 3$,

$$
\left\|\mathcal{Q}_{a, k}^{\ell}\right\|_{\mathbf{W}^{\alpha, 2}}^{2} \leq C^{k} B^{-(k-3)(3 / 4-\alpha / 4)}\|\mathrm{Q}\|_{L^{2}}\|\mathrm{Q}\|_{\mathbf{W}^{\beta, 2}}^{k-1}
$$

where $\ell=1,2, \alpha<\beta+1-\max \{0,1 / 4-\beta / 2\}$. In particular, $\sum_{k=3}^{\infty} \mathcal{Q}_{a, k}^{\ell}$ converges in $\mathbf{W}^{\alpha, 2}\left(\mathbb{R}^{2}\right)$, for $\alpha<\beta+3 / 4$, by choosing the constant $B$ large enough.

For the rest of the section we will assume that $n=2$.

It remains to estimate $\left\|\mathcal{Q}_{a, 2}^{\ell}\right\|_{\mathbf{W}^{\alpha, 2}}^{2}$. From (2.32) we can reduce this to the estimate the norm in $W^{\alpha, 2}\left(\mathbb{R}^{2}\right)$ of certain scalar functions.

To do this notice that each of the functions $\mathcal{Q}_{p, 2}^{1}, \mathcal{Q}_{s, 2}^{1}, \mathcal{Q}_{p, 2}^{2}$ and $\mathcal{Q}_{s, 2}^{2}$ can be written as linear combination of functions corresponding to longitudinal and transverse scattering amplitudes that we denote by $\mathcal{Q}_{p, 2}^{1, p}, \mathcal{Q}_{p, 2}^{1, s}$ and so on.

Given $a, b, c \in \mathbb{R}$ and $f, g$ complex functions in $\mathbb{R}^{2}$ we define $\mathcal{T}_{a c}^{b}(f, g)$ by

$$
\widehat{\mathcal{T}}_{a c}^{b}(f, g)(\omega \theta)=\left(f R\left(a^{2} \omega^{2}+i 0\right)\left(g(\cdot) e^{i b \omega \theta \cdot(\cdot)}\right)\right) \wedge(-c \omega \theta) .
$$

We find that to prove that $\mathcal{Q}_{a, 2}^{\ell} \in \mathbf{W}^{\alpha, 2}\left(\mathbb{R}^{2}\right)$, it suffices to see that $\mathcal{T}_{a c}^{b}\left(q_{1}, R_{k} R_{\ell} q_{2}\right)$ $\in W^{\alpha, 2}\left(\mathbb{R}^{2}\right)$ for $k, \ell \in\{1,2\}$, for any two of the entries $q_{1}, q_{2}$ of the matrix function $\mathrm{Q}$ and for certain values of the parameters $a, b$, and $c$. The following table contains all the values of the parameters $a, b$ and $c$ that appear in each of the eight cases to be studied. 


\begin{tabular}{|c||c|c||c|c||c|c||c|c|}
\hline & $\mathcal{Q}_{p, 2}^{1, p}$ & $\mathcal{Q}_{p, 2}^{1, s}$ & $\mathcal{Q}_{s, 2}^{1, p}$ & $\mathcal{Q}_{s, 2}^{1, s}$ & $\mathcal{Q}_{p, 2}^{2, s}$ & $\mathcal{Q}_{p, 2}^{2, p}$ & $\mathcal{Q}_{s, 2}^{2, s}$ & $\mathcal{Q}_{s, 2}^{2, p}$ \\
\hline \hline$a$ & 1 & $K$ & $\frac{2}{(K+1)}$ & $\frac{2 K}{(K+1)}$ & $\frac{2}{(K+1)}$ & $\frac{2 K}{(K+1)}$ & $K^{-1}$ & 1 \\
\hline$b$ & 1 & 1 & $\frac{2}{(K+1)}$ & $\frac{2}{(K+1)}$ & $\frac{2 K}{(K+1)}$ & $\frac{2 K}{(K+1)}$ & 1 & 1 \\
\hline$c$ & 1 & 1 & $\frac{2 K}{(K+1)}$ & $\frac{2 K}{(K+1)}$ & $\frac{2}{(K+1)}$ & $\frac{2}{(K+1)}$ & 1 & 1 \\
\hline
\end{tabular}

Rescaling the cases corresponding to $\mathcal{Q}_{s, 2}^{1, p}, \mathcal{Q}_{s, 2}^{1, s}, \mathcal{Q}_{p, 2}^{2, s}$ and $\mathcal{Q}_{p, 2}^{2, p}$ by making $2 \omega /(K+1)=\tilde{\omega}$, we have that it is enough to study the operators $\mathcal{T}_{a c}^{b}$ for the values of $a, b$ and $c$ given in the following table:

\begin{tabular}{|c||c|c|c|c|c|c|c|c|}
\hline$a$ & 1 & $K$ & 1 & $K$ & 1 & $K$ & $K^{-1}$ & 1 \\
\hline$b$ & 1 & 1 & 1 & 1 & $K$ & $K$ & 1 & 1 \\
\hline$c$ & 1 & 1 & $K$ & $K$ & 1 & 1 & 1 & 1 \\
\hline
\end{tabular}

The following proposition gives an expression of $\mathcal{T}_{a c}^{b}$ as the sum of a principal value and an integral operator over a sphere. Let us denote

$$
\Gamma_{r_{1}, r_{2}}(\eta)=\left\{\xi \in \mathbb{R}^{2}:\left|\xi-r_{1} \eta\right|=r_{2}|\eta|\right\} .
$$

Proposition 2.12. Given $\eta \in \mathbb{R}^{2}$,

$$
\widehat{\mathcal{T}}_{a c}^{b}(f, g)(\eta)=\widehat{\mathcal{G}}_{a c}^{b}(f, g)(\eta)+\widehat{\mathcal{H}}_{a c}^{b}(f, g)(\eta),
$$

where

$$
\widehat{\mathcal{G}}_{a c}^{b}(f, g)(\eta)=p \cdot v \cdot \int_{\mathbb{R}^{2}} \frac{\widehat{f}(\xi) \widehat{g}\left(\left(1+\frac{b}{c}\right) \eta-\xi\right)}{-|\eta-\xi|^{2}+\frac{a^{2}}{c^{2}}|\eta|^{2}} d \xi
$$

and

$$
\widehat{\mathcal{H}}_{a c}^{b}(f, g)(\eta)=\frac{i \pi c}{2 a|\eta|} \int_{\Gamma_{1, \frac{a}{c}}(\eta)} \widehat{f}(\xi) \widehat{g}\left(\left(1+\frac{b}{c}\right) \eta-\xi\right) d \sigma(\xi) .
$$

Proof. From (2.34) we can write

$$
\widehat{\mathcal{T}}_{a c}^{b}(f, g)(\omega \theta)=\left(\widehat{f} *\left(R\left(a^{2} \omega^{2}+i 0\right)\left(g(\cdot) e^{i b \omega \theta \cdot(\cdot)}\right)\right)\right)(-c \omega \theta) .
$$

The result follows making $-c \omega \theta=\eta$ and using the well-known identity

$$
R\left(k^{2}+i 0\right) g(x)=p . v . \int_{\mathbb{R}^{2}} \frac{1}{-|\xi|^{2}+k^{2}} \widehat{g}(\xi) e^{i x \cdot \xi} d \xi+\frac{i \pi}{2 k} \widehat{d \sigma_{k}} * g(x), \quad x \in \mathbb{R}^{2} .
$$

We introduce the following operators. 
Definition 2.13. Given $r_{1}, r_{2}>0$, for $\eta \in \mathbb{R}^{2}$ we define the operators $\mathcal{T}_{r_{1}, r_{2}}$ such that

$$
\widehat{\mathcal{T}}_{r_{1}, r_{2}}(f, g)(\eta)=\widehat{\mathcal{G}}_{r_{1}, r_{2}}(f, g)(\eta)+\widehat{\mathcal{H}}_{r_{1}, r_{2}}(f, g)(\eta),
$$

where

$$
\widehat{\mathcal{G}}_{r_{1}, r_{2}}(f, g)(\eta)=p . v \cdot \int_{\mathbb{R}^{2}} \frac{\widehat{f}(\xi) \widehat{g}(\eta-\xi)}{-\left|r_{1} \eta-\xi\right|^{2}+r_{2}^{2}|\eta|^{2}} d \xi
$$

and

$$
\widehat{\mathcal{H}}_{r_{1}, r_{2}}(f, g)(\eta)=\frac{i \pi}{2 r_{2}|\eta|} \int_{\Gamma_{r_{1}, r_{2}}(\eta)} \widehat{f}(\xi) \widehat{g}(\eta-\xi) d \sigma(\xi) .
$$

With this definition, we have that

$$
\widehat{\mathcal{T}}_{a c}^{b}(f, g)\left(\left(1+\frac{b}{c}\right)^{-1} \eta\right)= \begin{cases}\widehat{\mathcal{T}}_{r, r}(f, g)(\eta), & \text { if } a=c, \text { with } r=\left(1+\frac{b}{c}\right)^{-1}, \\ \widehat{\mathcal{T}}_{r, r}(g, f)(\eta), & \text { if } a=b, \text { with } r=\frac{b}{c}\left(1+\frac{b}{c}\right)^{-1}, \\ \widehat{\mathcal{T}}_{1 / 2, a / 2}(g, f)(\eta), & \text { if } b=c=1 .\end{cases}
$$

In Section 3 we will prove:

Proposition 2.14. Let $0<a, \beta<1$ and $b>0$. For $f, g \in W^{\beta, 2}\left(\mathbb{R}^{2}\right)$, the bilinear operator $\mathcal{H}_{a, b}$ satisfies

$$
\left\|\mathcal{H}_{a, b}(f, g)\right\|_{W^{\alpha, 2}} \leq C\|f\|_{W^{\beta, 2}}\|g\|_{W^{\beta, 2}},
$$

when $\alpha<\beta+1 / 2$.

Proposition 2.15. For $i=1,2$, let $q_{i}$ have compact support belonging to $L^{r}\left(\mathbb{R}^{2}\right) \cap$ $W^{\beta, 2}\left(\mathbb{R}^{2}\right)$, with $r>1$. Then $\mathcal{G}_{a, b}$ satisfies

$$
\mathcal{G}_{a, b}(f, g) \in W^{\alpha, 2}\left(\mathbb{R}^{2}\right)+\mathcal{C}^{\infty}\left(\mathbb{R}^{2}\right),
$$

and

$$
\left\|\mathcal{F}^{-1} \chi_{\{|\cdot|>1\}} \widehat{\mathcal{G}_{a, b}}(f, g)\right\|_{W^{\alpha, 2}} \leq C\left\|q_{1}\right\|_{W^{\beta, 2}}\left\|q_{2}\right\|_{W^{\beta, 2}},
$$

for $(f, g)=\left(q_{1}, q_{2}\right)$ or $\left(q_{1}, R_{j} R_{k} q_{2}\right), j, k=1,2$, if $\alpha<\beta+1 / 2$ and $\beta \in[0,1 / 2)$ and any $\alpha<1$ if $1 / 2 \leq \beta<1$.

From Propositions 2.14 and 2.15 we obtain:

Corollary 2.16. Let $n=2$ and consider the matrix $\mathrm{Q}$ in $\mathbf{W}^{\beta, 2}\left(\mathbb{R}^{2}\right)$ with compact support. If $0 \leq \beta<1, \ell=1,2$ and $a=p, s$, we have

$$
\left\|\mathcal{Q}_{a, 2}^{\ell}\right\|_{\mathbf{W}^{\alpha, 2}} \leq C\|\mathrm{Q}\|_{\mathbf{W}^{\beta, 2}}^{2},
$$

for $0<\alpha<1$ such that $\alpha<\beta+1 / 2$ if $\beta<1 / 2$, and any $\alpha<1$ if $\beta \geq 1 / 2$.

Proof of Theorem 1.1. The proof follows from Corollary 2.11 (which makes the difference between $\beta>1 / 2$ and $\beta<1 / 2$ ) and Propositions 2.14 and 2.15. 


\section{Smoothing properties of the operators $\mathcal{H}_{a, b}$ and $\mathcal{G}_{a, b}$}

In this section we will prove the following theorems.

Theorem 3.1. For $0<a, \alpha<1$ and $b>0$, let $2<l<3,1 /(2 l)+1 / p=1$. For $f, g \in W^{\alpha-1 / l, 2}\left(\mathbb{R}^{2}\right) \cap L^{p}\left(\mathbb{R}^{2}\right)$ we have

$$
\left\|\mathcal{H}_{a, b}(f, g)\right\|_{W^{\alpha, 2}} \leq A_{l}(a, b)\left\{\|f\|_{W^{\alpha-1 / l, 2}}\|g\|_{L^{p}}+\|f\|_{L^{p}}\|g\|_{W^{\alpha-1 / l, 2}}\right\} .
$$

Theorem 3.2. Let $2<l<3,0<\alpha, a<1,0<\alpha-1 / l, b>0$ and $q_{i}$ with compact support for $i=1,2$ and belonging to $W^{\alpha-1 / l, 2}\left(\mathbb{R}^{2}\right) \cap L^{r}\left(\mathbb{R}^{2}\right)$, for some $r>1$. Then for $(f, g)=\left(q_{1}, q_{2}\right)$ or $\left(q_{1}, R_{j} R_{k} q_{2}\right), j, k=1,2$,

$$
\mathcal{G}_{a, b}(f, g) \in W^{\alpha, 2}\left(\mathbb{R}^{2}\right)+\mathcal{C}^{\infty}\left(\mathbb{R}^{2}\right),
$$

and

$$
\left\|\mathcal{F}^{-1}\left(\chi_{\{|\cdot|>1\}} \widehat{\mathcal{G}}_{a, b}(f, g)\right)\right\|_{W^{\alpha, 2}} \leq C\left\|q_{1}\right\|_{W^{\alpha-1 / l, 2}}\left\|q_{2}\right\|_{W^{\alpha-1 / l, 2}},
$$

where $\mathcal{F}$ denotes the Fourier transform. The constant $C$ depends on $a, b$ and the supports of $q_{i}, i=1,2$.

Propositions 2.14 and 2.15 follow directly from these theorems.

\subsection{Proof of Theorem 3.1}

Since the spherical operator $\mathcal{H}_{1 / 2,1 / 2}$ was treated in [15], we will assume from now on that $(a, b) \neq(1 / 2,1 / 2)$.

Now we make some geometric considerations. Let

$$
\Phi=\left\{(\eta, \xi) \in \mathbb{R}^{2} \times \mathbb{R}^{2}:|\xi-a \eta|=b|\eta|\right\} .
$$

Then, for each $\eta, \Gamma_{a, b}(\eta)$ is the $\eta$-section of $\Phi$ :

$$
\Gamma_{a, b}(\eta)=\left\{\xi \in \mathbb{R}^{2}:(\eta, \xi) \in \Phi\right\} .
$$

For $\xi \in \mathbb{R}^{2}$ fixed and for $a \neq b$ we recognize the $\xi$-section of $\Phi$ as

$$
\Lambda_{a, b}(\xi)=\left\{\eta \in \mathbb{R}^{2}:|\xi-a \eta|=b|\eta|\right\}=\left\{\eta \in \mathbb{R}^{2}:\left|\eta-\frac{a}{a^{2}-b^{2}} \xi\right|=\frac{b|\xi|}{\left|a^{2}-b^{2}\right|}\right\}
$$

and for $a=b$,

$$
\Lambda_{a, b}(\xi)=\left\{\eta \in \mathbb{R}^{2}:\left\langle\xi, \frac{1}{2 a} \xi-\eta\right\rangle=0\right\} .
$$

Notice that

$$
\begin{aligned}
\widehat{\mathcal{H}}_{a, b}(f, g)(\eta) & =\frac{1}{b|\eta|} \int_{\Gamma_{a, b}(\eta)} \widehat{f}(\xi) \widehat{g}(\eta-\xi) d \sigma(\xi) \\
& =\frac{1}{b|\eta|} \int_{\Gamma_{a, b}^{+}(\eta)} \widehat{f}(\xi) \widehat{g}(\eta-\xi) d \sigma(\xi)+\frac{1}{b|\eta|} \int_{\Gamma_{a, b}^{-}(\eta)} \widehat{f}(\xi) \widehat{g}(\eta-\xi) d \sigma(\xi) \\
& =\widehat{\mathcal{H}}_{a, b}^{+}(f, g)(\eta)+\widehat{\mathcal{H}}_{a, b}^{-}(f, g)(\eta),
\end{aligned}
$$


where

$$
\begin{aligned}
& \Gamma_{a, b}(\eta)=\Gamma_{a, b}^{+}(\eta) \cup \Gamma_{a, b}^{-}(\eta), \\
& \Gamma_{a, b}^{+}(\eta)=\left\{\xi \in \mathbb{R}^{2}:|\xi-a \eta|=b|\eta|, \quad\langle\xi-a \eta, \eta\rangle \geq 0\right\} \\
& \Gamma_{a, b}^{-}(\eta)=\left\{\xi \in \mathbb{R}^{2}:|\xi-a \eta|=b|\eta|, \quad\langle\xi-a \eta, \eta\rangle \leq 0\right\} .
\end{aligned}
$$

Also define for $\xi \in \mathbb{R}^{2}$ the set $\Lambda_{a, b}^{+}(\xi)$ by

$$
\eta \in \Lambda_{a, b}^{+}(\xi) \Longleftrightarrow \xi \in \Gamma_{a, b}^{+}(\eta)
$$

We have that

$$
\mathcal{H}_{a, b}^{-}(f, g)(\eta)=\mathcal{H}_{1-a, b}^{+}(g, f)(\eta),
$$

and therefore we can reduce our study to the operator $\mathcal{H}_{a, b}^{+}(f, g)(\eta)$.

Now we describe the stereographic projection used in the paper. Let $\xi \neq 0$ in $\mathbb{R}^{2}$ and denote by $C_{r}(\xi)=\xi+r \mathbb{S}^{1}$ the circle centered at $\xi$ and radius $r>0$. Let $\ell$ be the line orthogonal to $\xi$ passing through $\xi-r e_{1}$, with $e_{1}=\xi /|\xi|$. For every $\zeta \in \ell$, let $\eta(\zeta)$ be the intersection of the line passing through $\zeta$ and the point $P_{1}=\xi+r e_{1}$. Then the mapping $\eta(\zeta)$ is a bijection of $\ell$ onto $C_{r}(\xi) \backslash\left\{P_{1}\right\}$.

Every $\zeta \in \ell$ can be written as $\zeta=(|\xi|-r) e_{1}+s e_{2} \in \ell$, with $e_{2}=e_{1}^{\perp}$ and $s \in \mathbb{R}$. Then,

$$
\eta(s)=\left(|\xi|+r-2 r\left(1+\left(\frac{s}{2 r}\right)^{2}\right)^{-1}\right) e_{1}+s\left(1+\left(\frac{s}{2 r}\right)^{2}\right)^{-1} e_{2},
$$

parametrizes $C_{r}(\xi) \backslash\left\{P_{1}\right\}$ in $\mathbb{R}$.

If $\eta \in C_{r}(\xi) \backslash\left\{P_{1}\right\}$ is written in polar coordinates as $\eta=(|\xi|+r \cos \theta) e_{1}+$ $r \sin \theta e_{2}, \theta \in(0,2 \pi)$, then $\eta=\eta(s)$ with

$$
s=\frac{2 r \sin \theta}{(1-\cos \theta)},
$$

where in particular the length measure in $C_{r}(\xi)$ in the variable $s$ is given by

$$
d \sigma=\left(1+\left(\frac{s}{2 r}\right)^{2}\right)^{-1} d s
$$

The following Fubini's theorem holds in $\Phi$ (see [15]):

Lemma 3.3. Let $h \geq 0$ be a measurable function on $\Phi$. Then, for $b>0$ and $-b \neq a \in \mathbb{R}$

$$
\int_{\mathbb{R}^{2}} \int_{\Gamma_{a, b}(\eta)} h(\xi, \eta) d \sigma_{\eta}(\xi) d \eta=\int_{\mathbb{R}^{2}} \int_{\Lambda_{a, b}(\xi)} h(\xi, \eta) \frac{|\eta|}{|\xi|} d \sigma_{\xi}(\eta) d \xi
$$

where $\sigma_{\zeta}$ will denote the Lebesgue measure on either $\Gamma_{a, b}(\zeta)$ or $\Lambda_{a, b}(\zeta)$, for $\zeta \in \mathbb{R}^{2}$.

To complete the proof of Theorem 3.1 we will need the following proposition. 
Proposition 3.4. Let $l>2, h \in L^{2 l}\left(\mathbb{R}^{2}\right)$ and

$$
F(\xi)=\int_{\Lambda_{a, b}^{+}(\xi)}\left(\int_{\Gamma_{a, b}^{+}(\eta)}\left|h\left(\eta-\xi^{\prime}\right)\right|^{2} d \sigma_{\eta}\left(\xi^{\prime}\right)\right)|\eta|^{2 \alpha-1} d \sigma_{\xi}(\eta) .
$$

We have

$$
F(\xi) \leq A_{l}^{2}(a, b)\|h\|_{L^{2 l}}^{2}|\xi|^{2 \alpha-2 / l+1} .
$$

Proof. Assume first that $a \neq b$.

We can split

$$
\Gamma_{a, b}^{+}(\eta)=\Gamma_{a, b}^{+, 1}(\eta) \cup \Gamma_{a, b}^{+, 2}(\eta),
$$

with

$$
\Gamma_{a, b}^{+, 1}(\eta)=\left\{\xi \in \mathbb{R}^{2}: \xi=a \eta+b \eta \cos \varphi+b \eta^{\perp} \sin \varphi, \quad 0 \leq \varphi \leq \pi / 2\right\},
$$

and a similar definition for $\Gamma_{a, b}^{+, 2}(\eta)$ with $-\pi / 2 \leq \varphi \leq 0$. For $\xi \in \mathbb{R}^{2}$ we introduce the set $\Lambda_{a, b}^{+, i}(\xi), i=1,2$, where

$$
\Lambda_{a, b}^{+}(\xi)=\Lambda_{a, b}^{+, 1}(\xi) \cup \Lambda_{a, b}^{+, 2}(\xi) \text { and } \eta \in \Lambda_{a, b}^{+, i}(\xi) \Longleftrightarrow \xi \in \Gamma_{a, b}^{+, i}(\eta) .
$$

Then

$$
F(\xi)=F_{1}(\xi)+F_{2}(\xi)
$$

with

$$
F_{i}(\xi)=\int_{\Lambda_{a, b}^{+, i}(\xi)}\left(\int_{\Gamma_{a, b}^{+, i}(\eta)}\left|h\left(\eta-\xi^{\prime}\right)\right|^{2} d \sigma_{\eta}\left(\xi^{\prime}\right)\right)|\eta|^{2 \alpha-1} d \sigma_{\xi}(\eta), \quad i=1,2 .
$$

It is not difficult to verify that

$$
\Lambda_{a, b}^{+, 1}(\xi)=\left\{\eta \in \mathbb{R}^{2}: \eta=\frac{a+b \cos \theta}{a^{2}-b^{2}} \xi+\frac{b}{a^{2}-b^{2}} \xi^{\perp} \sin \theta,-\pi \leq \theta \leq-\theta(a, b)\right\},
$$

(similar definition for $\Lambda_{a, b}^{+, 2}(\xi)$ with $\theta(a, b) \leq \theta \leq \pi$ ), where $\theta(a, b)$ is such that $\sin \theta(a, b)=\frac{a^{2}-b^{2}}{a^{2}+b^{2}}$ and $\cos \theta(a, b)=-\frac{2 a b}{a^{2}+b^{2}}$, and for $\eta=\eta(s)$,

$$
\Gamma_{a, b}^{+, 1}(\eta)=\left\{\xi^{\prime}(t)=\xi_{1}^{\prime}(t) \frac{\eta(s)}{|\eta(s)|}+\xi_{2}^{\prime}(t) \frac{\eta(s)^{\perp}}{|\eta(s)|}, 2 b|\eta(s)| \leq t<\infty\right\},
$$

(similar definition for $\Gamma_{a, b}^{+, 2}(\eta)$ with $\left.-\infty<t<-2 b|\eta(s)|\right)$ and

$$
\xi_{1}^{\prime}(t)=a|\eta(s)|+b|\eta(s)|-\frac{2 b|\eta(s)|}{1+\left(\frac{t}{2 b|\eta(s)|}\right)^{2}}, \quad \xi_{2}^{\prime}(t)=\frac{t}{1+\left(\frac{t}{2 b|\eta(s)|}\right)^{2}} .
$$

Hence, for $i=1,2$,

$$
F_{i}(\xi)=4 b^{2}|\xi| \iint_{D_{i}} \frac{|h(\eta(2 b|\xi| s)-\zeta(2 b|\eta(s)| t))|^{2}|\eta(2 b|\xi| s)|^{2 \alpha} d t d s}{\left(1+t^{2}\right)\left(1+\left(a^{2}-b^{2}\right)^{2} s^{2}\right)}
$$


where

$$
D_{1}=\left\{(s, t):-\frac{1}{(a+b)^{2}}<s<0, t>1\right\}, \quad D_{2}=\left\{(s, t): 0<s<\frac{1}{(a+b)^{2}}, t<0\right\} .
$$

A calculation gives us that

$$
\eta(2 b|\xi| s)-\xi^{\prime}(2 b|\eta(s)| t)=|\xi|\left(y_{1}(s, t) \mathbf{e}_{1}+y_{2}(s, t) \mathbf{e}_{2}\right),
$$

with

$$
\begin{gathered}
y_{1}(s, t)=\left(1-a-b+\frac{2 b}{1+t^{2}}\right) \tilde{\eta}_{1}(s)+\frac{2 b t}{1+t^{2}} \tilde{\eta}_{2}(s), \\
y_{2}(s, t)=\left(1-a-b+\frac{2 b}{1+t^{2}}\right) \tilde{\eta}_{2}(s)-\frac{2 b t}{1+t^{2}} \tilde{\eta}_{1}(s), \\
\tilde{\eta}_{1}(s)=\frac{1}{a^{2}-b^{2}}\left(a+b-\frac{2 b}{1+\left(a^{2}-b^{2}\right)^{2} s^{2}}\right) \quad \text { and } \quad \tilde{\eta}_{2}(s)=\frac{2 b s}{1+\left(a^{2}-b^{2}\right)^{2} s^{2}} .
\end{gathered}
$$

Let $\tilde{\eta}(s)=\left(\tilde{\eta}_{1}(s), \tilde{\eta}_{2}(s)\right)$ and $y(s, t)=\left(y_{1}(s, t) e_{1}+y_{2}(s, t) e_{2}\right)$. Then, from (3.3),

$$
F_{i}(\xi)=4 b^{2}|\xi|^{1+2 \alpha} \iint_{D_{i}} \mid h\left(\left.|\xi| y(s, t)\right|^{2} \frac{|\tilde{\eta}(s)|^{2 \alpha} d t d s}{\left(1+t^{2}\right)\left(1+\left(a^{2}-b^{2}\right)^{2} s^{2}\right)} .\right.
$$
bian

We consider the transformation $h(s, t)=|\xi| y(s, t)$, which for $b \neq a$ has jaco-

$$
J h(s, t)=\frac{8 b^{2}|\xi|^{2}|g(s, t)|}{\left(1+s^{2}\left(a^{2}-b^{2}\right)^{2}\right)^{2}\left(1+t^{2}\right)^{2}\left(a^{2}-b^{2}\right)},
$$

where

$$
\begin{aligned}
g(s, t)= & -(a+b)\left(a^{2}-b^{2}\right)^{2}(1-a) t s^{2}-(b-a)(1-a) t \\
& +\left(a^{2}-b^{2}\right)(1-a-b) a t^{2} s+\left|a^{2}-b^{2}\right|(a-b-1) a s .
\end{aligned}
$$

Let $l>2$ and $1 / l+1 / l^{\prime}=1$. By Hölder's inequality,

$$
F_{i}(\xi) \leq 4 b^{2}|\xi|^{1+2 \alpha}\|h\|_{L^{2 l}\left(\mathbb{R}^{2}\right)}^{2}\left(I_{i}(|\xi|, a, b)\right)^{1 / l^{\prime}},
$$

where

$$
I_{i}(|\xi|, a, b)=C \frac{\left|a^{2}-b^{2}\right|^{l^{\prime} / l}}{|\xi|^{2 l^{\prime} / l} b^{2 l^{\prime} / l}} \iint_{D_{i}} \frac{\left(1+\left(a^{2}-b^{2}\right)^{2} s^{2}\right)^{l^{\prime}-2}|\tilde{\eta}(s)|^{2 \alpha l^{\prime}} d t d s}{\left(1+t^{2}\right)^{2-l^{\prime}}|g(s, t)|^{l^{\prime} / l}}
$$

From $|\tilde{\eta}(s)|^{2} \leq \frac{2}{(a+b)^{2}}$ and $|g(s, t)|=|g(-s,-t)|$, we obtain

$$
\begin{aligned}
I_{i}(|\xi|, a, b) & \leq C \frac{\left|a^{2}-b^{2}\right|^{l^{\prime} / l}}{(a+b)^{2 \alpha l^{\prime}} b^{2 l^{\prime} / l}|\xi|^{2 l^{\prime} / l}} J(a, b), \\
J(a, b) & =\iint_{D_{1}} f(s, t) d s d t=\iint_{D_{2}} f(s, t) d s d t,
\end{aligned}
$$

with $f(s, t)=1 /\left(\left(1+t^{2}\right)^{2-l^{\prime}}\left(1+\left(a^{2}-b^{2}\right)^{2} s^{2}\right)^{2-l^{\prime}}|g(s, t)|^{l^{\prime} / l}\right)$. 
By the estimate of the integral $J(a, b)$ given in Proposition 4.1 in the appendix, the proposition follows in the case $a \neq b$.

For the case $a=b=r \neq 1 / 2$ we indicate briefly how to modify the previous proof: we have that

$$
\eta \in \Lambda_{r, r}^{+, i}(\xi) \Longleftrightarrow \eta=\eta(s)=\frac{|\xi|}{2 r} \mathbf{e}_{1}+s|\xi| \mathbf{e}_{2}
$$

where $s \in(-1 /(2 r), 0)$ for $i=1$, and $s \in(0,1 /(2 r))$ when $i=2$. Then (3.4) holds with $\tilde{\eta}(s)=\left(\tilde{\eta}_{1}(s), \tilde{\eta}_{2}(s)\right)=(1 /(2 r), 2 r s)$, hence $|\tilde{\eta}(s)| \leq 1 / 2 r^{2}$.

In this case,

(3.10) $J h(s, t)=\frac{4 r|\xi|^{2}|g(s, t)|}{\left(1+t^{2}\right)^{2}}$, where $g(s, t)=(1-r) t+2 r^{2} s\left[(1-2 r) t^{2}-1\right]$

With this in hand, we have that (3.6)-(3.9) hold for $a=b$, hence using the estimate of $J(r, r)$ in the appendix we complete the proof of the proposition.

Proof of Theorem 3.1. As mentioned, it suffices to estimate $\left\|\mathcal{H}_{a, b}^{+}(f, g)\right\|_{\dot{W}^{\alpha, 2}}^{2}$. By Lemma 3.3,

$$
\begin{aligned}
\| \mathcal{H}_{a, b}^{+} & (f, g) \|_{W^{\alpha, 2}}^{2}=\int_{\mathbb{R}^{2}}\left|\widehat{\mathcal{H}}_{a, b}^{+}(f, g)(\eta)\right|^{2}|\eta|^{2 \alpha} d \eta \\
& \leq \frac{1}{b^{2}} \int_{\mathbb{R}^{2}} \frac{1}{|\eta|^{2}}\left(\int_{\Gamma_{a, b}^{+}(\eta)}|\widehat{f}(\xi)||\widehat{g}(\eta-\xi)| d \sigma_{\eta}(\xi)\right)^{2}|\eta|^{2 \alpha} d \eta \\
& \leq \frac{1}{b^{2}} \int_{\mathbb{R}^{2}} \int_{\Gamma_{a, b}^{+}(\eta)}|\widehat{f}(\xi)|^{2}\left(\int_{\Gamma_{a, b}^{+}(\eta)}\left|\widehat{g}\left(\eta-\xi^{\prime}\right)\right|^{2} d \sigma_{\eta}\left(\xi^{\prime}\right)\right)|\eta|^{2 \alpha-2} d \sigma_{\eta}(\xi) d \eta \\
& \leq \frac{1}{b^{2}} \int_{\mathbb{R}^{2}}|\widehat{f}(\xi)|^{2} \int_{\Lambda_{a, b}^{+}(\xi)}\left(\int_{\Gamma_{a, b}^{+}(\eta)}\left|\widehat{g}\left(\eta-\xi^{\prime}\right)\right|^{2} d \sigma_{\eta}\left(\xi^{\prime}\right)\right) \frac{|\eta|^{2 \alpha-1}}{|\xi|} d \sigma_{\xi}(\eta) d \xi \\
& =\frac{1}{b^{2}} \int_{\mathbb{R}^{2}}|\widehat{f}(\xi)|^{2} F(\xi) \frac{d \xi}{|\xi|} .
\end{aligned}
$$

The theorem follows from Proposition 3.4, using the Hausdorff-Young inequality to estimate $\|\widehat{g}\|_{2 l} \leq\|g\|_{p}$.

Remark 3.5. The constant $A_{l}(a, b)$ in Theorem 3.1 was given in terms of the estimate for the function $J(a, b)$ proved in Proposition 4.1 in the appendix. By inspection one can see that with this construction $A_{l}(a, \cdot)$ is locally integrable in $(0, \infty)$, see Remark 4.3 .

Remark 3.6. We notice that Theorem 3.1 along with Remark 3.5 holds for the operator

$$
\widehat{\mathbf{H}}_{a, b}(f, g)(\eta)=\frac{1}{b|\eta|} \int_{\Gamma_{a, b}(\eta)}|\widehat{f}(\xi)||\widehat{g}(\eta-\xi)| d \sigma(\xi)
$$




\subsection{Proof of Theorem 3.2}

To study $\mathcal{G}_{a, b}$ we will use the following properties of the Sobolev spaces.

Lemma 3.7. If $g$ has compact support,

1) Given $d>0$, there exists a constant $C>0$, such that if $\xi$ and $\xi^{\prime}$ are in $\mathbb{R}^{2}$ and satisfy $\left|\xi-\xi^{\prime}\right| \leq d$, then

$$
|\hat{g}(\xi)| \leq C M \hat{g}\left(\xi^{\prime}\right)
$$

2) For $\beta \in \mathbb{R}, \gamma>0$, there exists a constant depending on $\beta, \gamma$ and the support of $g$ such that

$$
\|g\|_{\dot{W}^{\beta-\gamma, 2}} \leq C\|g\|_{\dot{W}^{\beta, 2}} .
$$

3) If $|\alpha|<1$ we have, for $i, k=1,2$,

$$
\begin{array}{r}
\left\|\mathcal{F}^{-1}(M \nabla \hat{g})\right\|_{\dot{W}^{\alpha, 2}} \leq C\|g\|_{\dot{W}^{\alpha, 2}}, \\
\left\|\mathcal{F}^{-1}\left(M \widehat{R_{j} R_{k} g}\right)\right\|_{\dot{W}^{\alpha, 2}} \leq C\|g\|_{\dot{W}^{\alpha, 2}}, \\
\left\|\mathcal{F}^{-1}\left(M \nabla \widehat{R_{j} R_{k} g}\right)\right\|_{\dot{W}^{\alpha, 2}} \leq C\|g\|_{W^{\alpha, 2}} .
\end{array}
$$

Proof. The proof of (3.11) and (3.12) for $0<\gamma<1$ can be found in [15]; then (3.12) for $\gamma>0$ can be obtained by iteration. For (3.13) see [12]. To prove (3.14) just notice that since $-1<\alpha<1$ then $|x|^{2 \alpha}$ is an $A_{2}$ weight in $\mathbb{R}^{2}$ (see [17]), so that $M$ is bounded in $L^{2}\left(\mathbb{R}^{2},|x|^{2 \alpha} d x\right)$. For (3.15) we use the same argument and

$$
\left|\partial_{i} \widehat{R_{j} R_{k}} g(\xi)\right| \leq C\left(\frac{|\widehat{g}(\xi)|}{|\xi|}+\left|\partial_{i} \widehat{g}(\xi)\right|\right), \quad \xi \in \mathbb{R}^{2}
$$

so that

$$
\begin{aligned}
& \|\left.\mathcal{F}^{-1}\left(M \nabla \widehat{R_{j} R_{k} g}\right)\right|_{\dot{W}^{\alpha, 2}} ^{2} \leq C \sum_{i=1}^{2} \int_{\mathbb{R}^{n}}\left|\partial_{i} \widehat{R_{j} R_{k} g}(\eta)\right|^{2}|\eta|^{2 \alpha} d \eta \\
& \leq C \int_{\mathbb{R}^{2}}|\widehat{g}(\eta)|^{2}|\eta|^{2(\alpha-1)} d \eta+C \sum_{i=1}^{2} \int_{\mathbb{R}^{2}}\left|\partial_{i} \widehat{g}(\eta)\right|^{2}|\eta|^{2 \alpha} d \eta
\end{aligned}
$$

Since $g$ has compact support there exists $\varphi \in C_{c}^{\infty}\left(\mathbb{R}^{2}\right)$ such that $\partial_{i} \widehat{g}=\widehat{\varphi g}=$ $\widehat{\varphi} * \widehat{g}$. Then $\left|\partial_{i} \widehat{g}(\eta)\right||\eta|^{\alpha} \leq C_{\beta}(1+|\cdot|)^{\alpha}|\widehat{\varphi}| *(1+|\cdot|)^{\alpha}|\widehat{g}|$, hence

$$
\int_{\mathbb{R}^{2}}\left|\partial_{i} \widehat{g}(\eta)\right|^{2}|\eta|^{2 \alpha} d \eta \leq C\|g\|_{W^{\alpha, 2}}^{2}
$$

Thus, from (3.16) we obtain (3.15).

We will also use Calderón's pointwise inequality for Sobolev functions (which is a mean value type theorem (see [4])). 
Lemma 3.8. Let $g \in \dot{W}^{1, p}\left(\mathbb{R}^{2}\right)=\left\{h \in \mathcal{S}^{\prime}\left(\mathbb{R}^{2}\right): \nabla h \in L^{p}\left(\mathbb{R}^{2}\right)\right\}$ for some $p>1$. Then we have, almost everywhere,

$$
|g(x)-g(y)| \leq C(M(\nabla g)(x)+M(\nabla g)(y))|x-y| .
$$

We notice that since $\mathcal{G}_{a, b}(f, g)=\mathcal{G}_{1-a, b}(g, f)$, then excluding the case $\mathcal{G}_{1 / 2,1 / 2}$ that was studied in ([15]), for the proof of Theorem 3.2 it will be sufficient to study $\mathcal{G}_{a, b}(f, g)$ when $(f, g)=\left(q_{1}, q_{2}\right),\left(q_{1}, R_{j} R_{k} q_{2}\right)$ and $\left(R_{j} R_{k} q_{1}, q_{2}\right)$ in the case $a \neq b$. Assume for the rest of the section that $a \neq b$.

To deal with the integral $\widehat{\mathcal{G}}_{a, b}(f, g)$, the idea is to split $\mathbb{R}^{2}$ for fixed $\eta$ in disjoint regions, one of them is a small annulus $M_{a, b}(\eta)$ containing the singularity on $|\xi-a \eta|=b|\eta|$. In order to bound the integral in this region we will use the cancelation of the kernel in (3.20) together with Lemma 3.8. The rest of the regions are dyadic annuli approaching the singularity. Harmonic analysis tools will be used to control the part of the integral over the annuli distant to the singularity, and the integral over the remaining parts will be estimated by integrating on spheres and using the estimates of the constants $A_{l}(a, s)$ appearing in the control of the norm of $\mathcal{H}_{a, s}(f, g)$ in Theorem 3.1.

Recalling that $a, b>0$, we let

$$
c_{1}=\frac{1}{2} \min \{a, b\}, c_{2}=\frac{3}{2} \max \{a, b\} \quad \text { and } d= \begin{cases}\frac{1}{4} \min \{b,|b-a|\}, & a \neq b, \\ \frac{1}{2} \min \{1, a\}, & a=b .\end{cases}
$$

For $\eta \in \mathbb{R}^{2}$, we define

$$
\begin{aligned}
A_{0}(\eta) & =\left\{\xi \in \mathbb{R}^{2}:|\xi-a \eta| \leq c_{1}|\eta|\right\}, \\
A_{j}(\eta) & =\left\{\xi:\left(1-\frac{1}{2^{j}}\right)\left(b-c_{1}\right)|\eta|<|\xi-a \eta|-c_{1}|\eta| \leq\left(1-\frac{1}{2^{j+1}}\right)\left(b-c_{1}\right)|\eta|\right\}, \\
j & =1,2, \ldots, N_{1}(\eta), \text { and } N_{1}(\eta)=\log _{2} \frac{\left(b-c_{1}\right)|\eta|}{2 d}, \\
M_{a, b}(\eta) & =\left\{\xi \in \mathbb{R}^{2}:|| \xi-a \eta|-b| \eta|| \leq d\right\}, \\
B_{i}(\eta) & =\left\{\xi \in \mathbb{R}^{2}: \frac{\left(c_{2}-b\right)}{2^{i}}|\eta|<|\xi-a \eta|-b|\eta| \leq \frac{\left(c_{2}-b\right)}{2^{i-1}}|\eta|\right\}, \\
i & =1,2, \ldots, N_{2}(\eta), \text { and } N_{2}(\eta)=\log _{2} \frac{\left(c_{2}-b\right)|\eta|}{d}, \text { and } \\
B_{0}(\eta) & =\left\{\xi \in \mathbb{R}^{2}:|\xi-a \eta| \geq c_{2}|\eta|\right\} .
\end{aligned}
$$

We decompose the operator $\widehat{\mathcal{G}}_{a, b}$ as

$$
\widehat{\mathcal{G}}_{a, b}=\widehat{\mathcal{P}}_{0}+\sum_{j=1}^{N_{1}(\eta)} \widehat{\mathcal{P}}_{j}+\widehat{\mathcal{Q}}_{0}+\sum_{i=1}^{N_{2}(\eta)} \widehat{\mathcal{Q}}_{i}+\widehat{\mathcal{V}}
$$


where

$$
\begin{aligned}
\widehat{\mathcal{V}}(f, g)(\eta) & =p \cdot v \cdot \int_{M_{a, b}(\eta)} \frac{\hat{f}(\xi) \hat{g}(\eta-\xi)}{|\xi-a \eta|^{2}-b^{2}|\eta|^{2}} d \xi \\
\widehat{\mathcal{P}}_{j}(f, g)(\eta) & =\int_{A_{j}(\eta)} \frac{\hat{f}(\xi) \hat{g}(\eta-\xi)}{b^{2}|\eta|^{2}-|\xi-a \eta|^{2}} d \xi, \quad j=0,1,2, \ldots, N_{1}(\eta), \\
\widehat{\mathcal{Q}}_{i}(f, g)(\eta) & =\int_{B_{i}(\eta)} \frac{\hat{f}(\xi) \hat{g}(\eta-\xi)}{|\xi-a \eta|^{2}-b^{2}|\eta|^{2}} d \xi, \quad i=0,1,2, \ldots, N_{2}(\eta) .
\end{aligned}
$$

The proof of Theorem 3.2 will be an immediate consequence of the following two results.

Proposition 3.9. Let $0<\alpha<1$ and $l>2$ such that

$$
0<\alpha-\frac{1}{l}
$$

If $q_{1}$ and $q_{2}$ have compact support in $W^{\alpha-1 / l, 2}\left(\mathbb{R}^{2}\right)$ and $(f, g)=\left(q_{1}, q_{2}\right)$ or $(f, g)=$ $\left(q_{1}, R_{j} R_{k} q_{2}\right)$, then

$$
\|\mathcal{G}(f, g)-\mathcal{V}(f, g)\|_{W^{\alpha, 2}} \leq C\left\|q_{1}\right\|_{W^{\alpha-1 / l, 2}}\left\|q_{2}\right\|_{W^{\alpha-1 / l, 2}},
$$

where the constant $C>0$ depends on $a, b$, and the supports of the $q_{1}$ and $q_{2}$.

Proposition 3.10. Let $\alpha<1$ and $l>2$ such that $0 \leq \alpha-1 / l$. If $q_{1}$ and $q_{2}$ have compact support and belong to $L^{r}\left(\mathbb{R}^{2}\right)$ for some $r>1$ and $(f, g)=\left(R_{j} q_{1}, q_{2}\right)$ or $(f, g)=\left(q_{1}, R_{j} q_{2}\right)$, then

$$
\left\|\mathcal{F}^{-1}\left(\chi_{\{|\cdot|>1\}}(\cdot) \widehat{\mathcal{V}}(f, g)(\cdot)\right)\right\|_{W^{\alpha, 2}} \leq C\left\|q_{1}\right\|_{W^{\alpha-1 / l, 2}}\left\|q_{2}\right\|_{W^{\alpha-1 / l, 2}},
$$

where the constant $C>0$ depends on $a, b$, and the supports of $q_{1}$ and $q_{2}$.

We will prove Proposition 3.9 by analyzing each part in (3.19) except the operator $\widehat{\mathcal{V}}$. We start by studying the boundedness of the operators $P_{0}$ and $Q_{0}$.

Proposition 3.11. If $q_{1}$ and $q_{2}$ have compact support in $W^{\alpha-1 / l, 2}\left(\mathbb{R}^{2}\right)$, then for any of the cases $(f, g)=\left(q_{1}, q_{2}\right),(f, g)=\left(q_{1}, R_{j} R_{k} q_{2}\right)$ or $(f, g)=\left(R_{j} R_{k} q_{1}, q_{2}\right)$ we have

(1) If $0 \leq \alpha<1$ and $l>2$,

$$
\left\|\mathcal{P}_{0}(f, g)\right\|_{W^{\alpha, 2}} \leq C\left\|q_{1}\right\|_{W^{\alpha-1 / l, 2}}\left\|q_{2}\right\|_{W^{\alpha-1 / l, 2}}
$$

(2) If $\alpha-1 / l>0$,

$$
\left\|\mathcal{Q}_{0}(f, g)\right\|_{W^{\alpha, 2}} \leq C\left\|q_{1}\right\|_{W^{\alpha-1 / l, 2}}\left\|q_{2}\right\|_{W^{\alpha-1 / l, 2}} .
$$

The constant $C>0$ depends on $a, b$, and the supports of $q_{1}$ and $q_{2}$. 
Proof. (1) Since $\left|\widehat{R_{j} R_{k} q_{1}}(\xi)\right| \leq\left|\widehat{q_{1}}(\xi)\right|$, then

$\left|\widehat{\mathcal{P}}_{0}\left(R_{j} R_{k} q_{1}, q_{2}\right)(\eta)\right| \leq \int_{A_{0}(\eta)} \frac{\left|\widehat{q_{1}}(\xi)\right|\left|\widehat{q_{2}}(\eta-\xi)\right|}{|a \eta-\xi|^{2}-|\eta|^{2}} d \xi \leq \frac{C}{|\eta|^{2}} \int_{A_{0}(\eta)}\left|\widehat{q_{1}}(\xi)\right|\left|\widehat{q_{2}}(\eta-\xi)\right| d \xi$.

If $\xi \in A_{0}(\eta)$ then $|\xi| \leq\left(a+c_{1}\right)|\eta|$. Hence, by (3.12), if $\varepsilon>0$ is such that $\alpha-1+\varepsilon<0$,

$$
\begin{aligned}
\left\|\mathcal{P}_{0}\left(R_{j} R_{k} q_{1}, q_{2}\right)\right\|_{\dot{W}^{\alpha, 2}}^{2} & \leq\left\|q_{2}\right\|_{\dot{W}^{-\varepsilon, 2}}^{2} \int_{\mathbb{R}^{2}} C|\eta|^{2 \alpha-4+2 \varepsilon} \int_{0<|\xi| \leq\left(1+a+c_{1}\right)|\eta|}\left|\widehat{q_{1}}(\xi)\right|^{2} d \xi \\
& \leq\left\|q_{2}\right\|_{\dot{W}^{-\varepsilon, 2}}^{2} \int_{\mathbb{R}^{2}}\left|\widehat{q_{1}}(\xi)\right|^{2} \int_{0<|\xi| \leq\left(1+a+c_{1}\right)|\eta|} C|\eta|^{2 \alpha-4+2 \varepsilon} d \eta d \xi \\
& \leq C\left\|q_{1}\right\|_{\dot{W}^{\alpha-1+\varepsilon, 2}}^{2}\left\|q_{2}\right\|_{\dot{W}^{-\varepsilon, 2}}^{2} \leq C\left\|q_{1}\right\|_{W^{\alpha-1 / l}}^{2}\left\|q_{2}\right\|_{\dot{W}^{\alpha-1 / l, 2}}^{2} .
\end{aligned}
$$

Since this is also true for $\alpha=0$, we obtain (3.21).

The estimate of $\left\|P_{0}\left(q_{1}, q_{2}\right)\right\|_{W^{\alpha, 2}}$ is included in the previous case and $\| P_{0}\left(q_{1}\right.$, $\left.R_{j} R_{k} q_{2}\right) \|_{W^{\alpha, 2}}$ can be treated in a similar way.

(2) Let $\beta$ such that $1-\beta<\alpha$.

Notice that if $\xi \in B_{0}(\eta)$, then $|\xi-a \eta| \geq C|\xi-\eta|$ and

$$
(|\xi-a \eta|+b|\eta|)(|\xi-a \eta|-b|\eta|) \geq C|\xi-\eta|^{\beta}|\eta|^{2-\beta} .
$$

Hence

$$
\begin{aligned}
\left|\widehat{\mathcal{Q}}_{0}\left(R_{j} R_{k} q_{1}, q_{2}\right)(\eta)\right| & \leq \int_{B_{0}(\eta)} \frac{\left|\widehat{q_{1}}(\xi)\right|\left|\widehat{q_{2}}(\eta-\xi)\right|}{(|\xi-a \eta|+b|\eta|)(|\xi-a \eta|-b|\eta|)} d \xi \\
& \leq \frac{C}{|\eta|^{2-\beta}} \int_{B_{0}(\eta)}\left|\widehat{q_{1}}(\xi)\right| \frac{\left|\widehat{q_{2}}(\eta-\xi)\right|}{|\xi-\eta|^{\beta}} d \xi
\end{aligned}
$$

and

$$
\begin{aligned}
\| \mathcal{Q}_{0}\left(R_{j}\right. & \left.R_{k} q_{1}, q_{2}\right) \|_{\dot{W}^{\alpha, 2}}^{2} \leq C \int_{\mathbb{R}^{2}}|\eta|^{2 \alpha-4+2 \beta}\left(\int_{B_{0}(\eta)}\left|\widehat{q_{1}}(\xi)\right| \frac{\left|\widehat{q_{2}}(\eta-\xi)\right|}{|\xi-\eta|^{\beta}} d \xi\right)^{2} d \eta \\
& \leq C \int_{\mathbb{R}^{2}}|\eta|^{2 \alpha-4+2 \beta} \int_{B_{0}(\eta)}\left|\widehat{q_{1}}(\xi)\right|^{2} d \xi\left(\int_{\mathbb{R}^{2}} \frac{\left|\widehat{q_{1}}(\eta-\zeta)\right|^{2}}{|\eta-\zeta|^{2 \beta}} d \zeta\right) d \eta \\
& \leq C\left\|q_{2}\right\|_{\dot{W}^{-\beta, 2}}^{2} \int_{\mathbb{R}^{2}}\left|\widehat{q_{1}}(\xi)\right|^{2} \int_{|\eta| \leq \frac{2}{|a|}|\xi|}|\eta|^{2 \alpha-4+2 \beta} d \eta d \xi \\
& \leq C\left\|q_{2}\right\|_{\dot{W}^{-\beta, 2}}^{2} \int_{\mathbb{R}^{2}}\left|\widehat{q_{1}}(\xi)\right|^{2}|\xi|^{2 \alpha-2+2 \beta} d \xi \leq C\left\|q_{1}\right\|_{\dot{W}^{\alpha+\beta-1}}^{2}\left\|q_{2}\right\|_{\dot{W}^{-\beta}}^{2} .
\end{aligned}
$$

Let $\beta=1-1 / l$, then using again (3.12) it follows that

$$
\left\|\mathcal{Q}_{0}\left(R_{j} R_{k} q_{1}, q_{2}\right)\right\|_{\dot{W}^{\alpha, 2}} \leq C\left\|q_{1}\right\|_{W^{\alpha-1 / l, 2}}\left\|q_{2}\right\|_{W^{\alpha-1 / l, 2}} .
$$

Now if we use (3.23) for $\alpha=0$ and $\beta>1$ we obtain

$$
\left\|\mathcal{Q}_{0}\left(R_{j} R_{k} q_{1}, q_{2}\right)\right\|_{L^{2}} \leq C\left\|q_{1}\right\|_{W^{\beta-1,2}}\left\|q_{2}\right\|_{W^{-\beta, 2}} .
$$


Notice that if $1-\beta+\alpha-1 / l \geq 0$, then from (3.12),

$$
\begin{aligned}
\left\|q_{1}\right\|_{W^{\beta-1,2}} & \leq C\left(\left\|q_{1}\right\|_{L^{2}}+\left\|q_{1}\right\|_{\dot{W}^{\alpha-1 / l-(1-\beta+\alpha-1 / l), 2}}\right) \\
& \leq C\left(\left\|q_{1}\right\|_{L^{2}}+\left\|q_{1}\right\|_{\dot{W}^{\alpha-1 / l, 2}}\right) \leq C\left\|q_{1}\right\|_{W^{\alpha-1 / l, 2}} .
\end{aligned}
$$

On the other hand, if $\beta+\alpha-1 / l \geq 0$, then

$$
\begin{aligned}
\left\|q_{2}\right\|_{W-\beta, 2} & \leq C\left(\left\|q_{2}\right\|_{L^{2}}+\left\|q_{2}\right\|_{\dot{W}^{\alpha-1 / l-(\beta+\alpha-1 / l), 2}}\right) \\
& \leq C\left(\left\|q_{2}\right\|_{L^{2}}+\left\|q_{2}\right\|_{\dot{W}^{\alpha-1 / l, 2}}\right) \leq C\left\|q_{2}\right\|_{W^{\alpha-1 / l, 2}} .
\end{aligned}
$$

Since $\alpha-1 / l>0$, we can choose $\beta>1$ such that

$$
-\alpha+\frac{1}{l} \leq \beta \leq 1+\alpha-\frac{1}{l} .
$$

Then (3.22) follows from (3.23)-(3.27).

The estimates corresponding to the pair $\left(q_{1}, R_{j} R_{k} q_{2}\right)$ and $\left(q_{1}, q_{2}\right)$ are similar.

Now we study the operators $\sum_{i=1}^{N_{2}(\eta)} Q_{i}$ and $\sum_{j=1}^{N_{1}(\eta)} P_{i}$.

Proposition 3.12. Let $a \in(0,1), b>0,2<l<3,1 /(2 l)+1 / p=1$ and $\alpha<1$. For any of the cases $(f, g)=\left(q_{1}, q_{2}\right),(f, g)=\left(q_{1}, R_{j} R_{k} q_{2}\right)$ or $(f, g)=$ $\left(R_{j} R_{k} q_{1}, q_{2}\right)$, with $q_{1}$ and $q_{2}$ with compact support in $W^{\alpha-1 / l, 2}\left(\mathbb{R}^{2}\right)$, we have

$$
\begin{gathered}
\left\|\sum_{i=1}^{N_{2}(\eta)} \mathcal{Q}_{i}(f, g)\right\|_{W^{\alpha, 2}} \leq C\left\|q_{1}\right\|_{W^{\alpha-1 / l, 2}}\left\|q_{2}\right\|_{W^{\alpha-1 / l, 2}}, \\
\left\|\sum_{j=1}^{N_{1}(\eta)} \mathcal{P}_{j}(f, g)\right\|_{W^{\alpha, 2}} \leq C\left\|q_{1}\right\|_{W^{\alpha-1 / l, 2}}\left\|q_{2}\right\|_{W^{\alpha-1 / l, 2}},
\end{gathered}
$$

where the constant $C$ may depend on $a, b$, and the supports of $q_{1}$ and $q_{2}$.

Proof. We start by proving (3.28). We can write, for $i=1,2, \ldots, N_{2}(\eta)$,

$$
B_{i}(\eta)=\left\{\xi \in \mathbb{R}^{2}: \xi=a \eta+\zeta \text { with } \zeta \in \Gamma_{a, b+r\left(c_{2}-b\right)}(\eta), \frac{1}{2^{i}}<r \leq \frac{1}{2^{i-1}}\right\} .
$$

Then,

$$
\begin{aligned}
& \left|\widehat{\mathcal{Q}}_{i}(f, g)(\eta)\right| \leq \int_{B_{i}(\eta)} \frac{\left|\widehat{q_{1}}(\xi)\right|\left|\widehat{q_{2}}(\eta-\xi)\right|}{|\xi-a \eta|^{2}-b^{2}|\eta|^{2}} d \xi \\
& =\int_{\frac{1}{2^{i}}<r \leq \frac{1}{2^{i}-1}} \int_{\Gamma_{a, b+r\left(c_{2}-b\right)}(\eta)} \frac{\left|\widehat{q_{1}}(\xi)\right|\left|\widehat{q_{2}}(\eta-\xi)\right|}{|\xi-a \eta|^{2}-b^{2}|\eta|^{2}} d \sigma_{b+r\left(c_{2}-b\right)}(\xi) d r \\
& =\int_{\frac{1}{2^{i}}}^{\frac{1}{2^{i-1}}} \frac{1}{\left(2 r\left(c_{2}-b\right)+r^{2}\left(c_{2}-b\right)^{2}\right)|\eta|^{2}} \int_{\Gamma_{a, b+r\left(c_{2}-b\right)}(\eta)}\left|\widehat{q_{1}}(\xi)\right|\left|\widehat{q_{2}}(\eta-\xi)\right| d \sigma_{b+r\left(c_{2}-b\right)}(\xi) d r \\
& =\frac{1}{|\eta|} \int_{\frac{1}{2^{i}}}^{\frac{1}{2^{i-1}}} \frac{b+r\left(c_{2}-b\right)}{\left(2 r\left(c_{2}-b\right)+r^{2}\left(c_{2}-b\right)^{2}\right)} \widehat{\mathbf{H}}_{a, b+r\left(c_{2}-b\right)}\left(q_{1}, q_{2}\right)(\eta) d r .
\end{aligned}
$$


Since $\frac{b+r\left(c_{2}-b\right)}{2\left(c_{2}-b\right)+r\left(c_{2}-b\right)^{2}} \leq 1$,

$$
\begin{aligned}
& \left|\sum_{i=1}^{N_{2}(\eta)} \widehat{\mathcal{Q}}_{i}(f, g)(\eta)\right| \leq \frac{1}{|\eta|} \sum_{i=1}^{N_{2}(\eta)} 2^{i} \int_{\frac{1}{2^{i}}}^{\frac{1}{2^{i-1}}} \widehat{\mathbf{H}}_{a, b+r\left(c_{2}-b\right)}\left(q_{1}, q_{2}\right)(\eta) d r \\
& \quad \leq \frac{2^{N_{2}(\eta)}}{|\eta|} \int_{\frac{1}{2^{N_{2}(\eta)}}}^{1} \widehat{\mathbf{H}}_{a, b+r\left(c_{2}-b\right)}\left(q_{1}, q_{2}\right)(\eta) d r \leq C \int_{0}^{1}\left|\widehat{\mathbf{H}}_{a, b+r\left(c_{2}-b\right)}\left(q_{1}, q_{2}\right)(\eta)\right| d r .
\end{aligned}
$$

By Minkowski's inequality we have

$$
\begin{aligned}
& \left\|\sum_{i=1}^{N_{2}(\cdot)} \mathcal{Q}_{i}(f, g)\right\|_{\dot{W}^{\alpha, 2}}=\left\|\sum_{i=1}^{N_{2}(\cdot)} \widehat{\mathcal{Q}}_{i}(f, g)(\cdot)|\cdot|^{\alpha}\right\|_{L^{2}} \\
& \quad \leq C\left\|\int_{0}^{1} \widehat{\mathbf{H}}_{a, b+r\left(c_{2}-b\right)}\left(q_{1}, q_{2}\right)(\cdot) d r|\cdot|^{\alpha}\right\|_{L^{2}} \\
& \quad \leq C \int_{0}^{1}\left\|\widehat{\mathbf{H}}_{a, b+r\left(c_{2}-b\right)}\left(q_{1}, q_{2}\right)(\cdot)|\cdot|^{\alpha}\right\|_{L^{2}} d r=C \int_{0}^{1}\left\|\mathbf{H}_{a, b+r\left(c_{2}-b\right)}\left(q_{1}, q_{2}\right)\right\|_{\dot{W}^{\alpha, 2}} d r .
\end{aligned}
$$

Theorem 3.1 and Remarks 3.5 and 3.6 yield

$$
\begin{aligned}
& \int_{0}^{1}\left\|\mathbf{H}_{a, b+r\left(c_{2}-b\right)}\left(q_{1}, q_{2}\right)\right\|_{\dot{W}^{\alpha, 2}} d r \\
& \quad \leq C\left\{\left\|q_{1}\right\|_{W^{\alpha-1 / l, 2}}\left\|q_{2}\right\|_{L^{p}}+\left\|q_{1}\right\|_{L^{p}}\left\|q_{2}\right\|_{W^{\alpha-1 / l, 2}}\right\} \int_{0}^{1} A_{l}\left(a, b+r\left(c_{2}-b\right)\right) d r \\
& \quad=C\left\{\left\|q_{1}\right\|_{W^{\alpha-1 / l, 2}}\left\|q_{2}\right\|_{L^{p}}+\left\|q_{1}\right\|_{L^{p}}\left\|q_{2}\right\|_{W^{\alpha-1 / l, 2}}\right\} .
\end{aligned}
$$

As $p<2$ and $q_{i}, i=1,2$, has compact support we get

$$
\left\|q_{i}\right\|_{L^{p}} \leq C\left\|q_{i}\right\|_{L^{2}} \leq C\left\|q_{i}\right\|_{W^{\alpha-1 / l, 2}}
$$

and

$$
\left\|\sum_{i=1}^{N_{2}(\cdot)} \mathcal{Q}_{i}(f, g)\right\|_{\dot{W}^{\alpha, 2}} \leq C\left\|q_{1}\right\|_{W^{\alpha-1 / l, 2}}\left\|q_{2}\right\|_{W^{\alpha-1 / l, 2} .}
$$

This estimation is valid for $\alpha=0$, so the proof of (3.28) is complete.

To prove (3.29) we consider $i_{0}$ such that $b-\frac{1}{2^{i_{0}-1}}\left(c_{2}-b\right)>0$. Then proceeding as in the proof of (3.28) we obtain that

$$
\left\|\sum_{j=i_{0}}^{N_{1}(\eta)} \mathcal{P}_{j}(f, g)\right\|_{W^{\alpha, 2}} \leq C(a, b)\left\|q_{1}\right\|_{W^{\alpha-1 / l, 2}}\left\|q_{2}\right\|_{W^{\alpha-1 / l, 2}} .
$$

Now it remains to deal with the first $i_{0}-1$ terms, but for $i>0$ fixed, the norms $\left\|P_{j}(f, g)\right\|_{W^{\alpha, 2}}$ can be handled as the case $i=0$ in Proposition 3.11.

Proof of Proposition 3.9. The proof follows from Propositions 3.11 and 3.12. 
For $0<\varepsilon<d$ we define

$M_{a, b}^{\varepsilon,-}(\eta)=\{\xi: \varepsilon<b|\eta|-|\xi-a \eta|<d\}, M_{a, b}^{\varepsilon,+}(\eta)=\{\xi: \varepsilon<|\xi-a \eta|-b|\eta|<d\}$,

so that

$\widehat{\mathcal{V}}(f, g)(\eta)=\lim _{\substack{\varepsilon \rightarrow 0 \\ \varepsilon>0}}\left(\int_{M_{a, b}^{\varepsilon,+}(\eta)} \frac{\hat{f}(\xi) \hat{g}(\eta-\xi)}{|\xi-a \eta|^{2}-b^{2}|\eta|^{2}} d \xi+\int_{M_{a, b}^{\varepsilon,-}(\eta)} \frac{\hat{f}(\xi) \hat{g}(\eta-\xi)}{|\xi-a \eta|^{2}-b^{2}|\eta|^{2}} d \xi\right)$.

For the proof of Proposition 3.10 we will use the following lemma.

Lemma 3.13. Let $T_{a, b}^{\varepsilon}$ be the operator defined by

$$
\widehat{T}_{a, b}^{\varepsilon}(f, g)(\eta)=\chi_{\{\eta:|\eta|>1\}}(\eta)|\eta|^{-1} \int_{M_{a, b}^{\varepsilon,+}(\eta) \cup M_{a, b}^{\varepsilon,-}(\eta)}|\widehat{f}(\xi)||\widehat{g}(\eta-\xi)| d \xi
$$

$\alpha \in \mathbb{R}$ and $l>2,1 /(2 l)+1 / p=1$. For $f, g \in W^{\alpha-1 / l, 2}\left(\mathbb{R}^{2}\right) \cap L^{p}\left(\mathbb{R}^{2}\right)$ we have

$$
\begin{aligned}
\left\|T_{a, b}^{\varepsilon}(f, g)\right\|_{\dot{W}^{\alpha, 2}} & \leq C(a, b)\left\{\|f\|_{\dot{W}^{\alpha-1 / l, 2}}\|\widehat{g}\|_{L^{2 l}}+\|\widehat{f}\|_{L^{2 l}}\|g\|_{\dot{W}^{\alpha-1 / l, 2}}\right\} \\
& \leq C(a, b)\left\{\|f\|_{\dot{W}^{\alpha-1 / l, 2}}\|g\|_{L^{p}}+\|f\|_{L^{p}}\|g\|_{\dot{W}^{\alpha-1 / l, 2}}\right\} .
\end{aligned}
$$

In particular, if we take $\alpha=0$ in (3.30) we have

$$
\begin{aligned}
\left\|T_{a, b}^{\varepsilon}(f, g)\right\|_{W^{\alpha, 2}} & \leq C(a, b)\left\{\|f\|_{W^{\alpha-1 / l, 2}}\|\widehat{g}\|_{L^{2 l}}+\|\widehat{f}\|_{L^{2 l}}\|g\|_{W^{\alpha-1 / l, 2}}\right\} \\
& \leq C(a, b)\left\{\|f\|_{W^{\alpha-1 / l, 2}}\|g\|_{L^{p}}+\|f\|_{L^{p}}\|g\|_{W^{\alpha-1 / l, 2}}\right\} .
\end{aligned}
$$

Proof. We rewrite $M_{a, b}^{\varepsilon,+}(\eta) \cup M_{a, b}^{\varepsilon,-}(\eta)$ as

$$
\left\{\xi \in \Gamma_{a, b s}(\eta): 1-\frac{d}{b|\eta|} \leq s \leq 1-\frac{\varepsilon}{b|\eta|}\right\} \bigcup\left\{\xi \in \Gamma_{a, b s}(\eta): 1+\frac{\varepsilon}{b|\eta|} \leq s \leq 1+\frac{d}{b|\eta|}\right\} .
$$

Then,

$$
\begin{aligned}
& \widehat{T}_{a, b}^{\varepsilon}(f, g)(\eta)=\widehat{T}_{a, b}^{\varepsilon,+}(f, g)(\eta)+\widehat{T}_{a, b}^{\varepsilon,-}(f, g)(\eta) \\
& \widehat{T}_{a, b}^{\varepsilon, \pm}(f, g)(\eta)=\chi_{\{\eta:|\eta|>1\}}(\eta)|\eta|^{-1} \int_{M_{a, b}^{\varepsilon, \pm}(\eta)}|\widehat{f}(\xi)||\widehat{g}(\eta-\xi)| d \xi
\end{aligned}
$$

We will study $\widehat{T}_{a, b}^{\varepsilon,-}(f, g)(\eta)$ :

$$
\begin{aligned}
& \widehat{T}_{a, b}^{\varepsilon,-}(f, g)(\eta)=\chi_{\{\eta:|\eta|>1\}}(\eta)|\eta|^{-1} \int_{1-\frac{d}{b|\eta|}}^{1-\frac{\varepsilon}{b|\eta|}} \int_{\Gamma_{a, b s}(\eta)}|\widehat{f}(\xi)||\widehat{g}(\eta-\xi)| d \sigma(\xi) d s \\
& \quad=b \chi_{\{\eta:|\eta|>1\}}(\eta) \int_{1-\frac{d}{b|\eta|}}^{1-\frac{\varepsilon}{b|\eta|}} s \widehat{\mathbf{H}}_{a, b s}(f, g)(\eta) d s \leq C(a, b) \int_{1-\frac{d}{b}}^{1} s \widehat{\mathbf{H}}_{a, b s}(f, g)(\eta) .
\end{aligned}
$$


Now we use Minkowski's inequality, Theorem 3.1 and (3.5) so that

$$
\begin{aligned}
\left\|T_{a, b}^{\varepsilon,-}(f, g)\right\|_{\dot{W}^{\alpha, 2}} & =\left\|\widehat{T}_{a, b}^{\varepsilon,-}(f, g)(\cdot)|\cdot|^{\alpha}\right\|_{L^{2}} \leq C\left\|\int_{1-\frac{d}{b}}^{1} s \widehat{\mathbf{H}}_{a, b s}(f, g)(\cdot) d s|\cdot|{ }^{\alpha}\right\|_{L^{2}} \\
& \leq C(a, b)\left\{\|f\|_{\dot{W}^{\alpha-1 / l, 2}}\|g\|_{L^{p}}+\|f\|_{L^{p}}\|f\|_{\dot{W}^{\alpha-1 / l, 2}}\right\} .
\end{aligned}
$$

Define

$$
\phi: M_{a, b}^{\varepsilon,-}(\eta) \rightarrow M_{a, b}^{\varepsilon,+}(\eta)
$$

a reflection with respect to $\Gamma_{a, b}(\eta)$ given by

$$
\phi(\xi)=a \eta+\left(2 \frac{b|\eta|}{|\xi-a \eta|}-1\right)(\xi-a \eta)=2 a \eta-\xi+2 b|\eta| \frac{\xi-a \eta}{|\xi-a \eta|}
$$

Then $\phi$ is a bijection, $\phi^{2}=I$ is the identity, and

$$
\begin{aligned}
\left|J_{\phi}(\xi)\right| & =1+2 \frac{b|\eta|-|\xi-a \eta|}{|\xi-a \eta|} . \\
|\phi(\xi)-a \eta|-b|\eta| & =b|\eta|-|\xi-a \eta|, \quad \xi \in M_{a, b}^{\varepsilon,-}(\eta) . \\
|\phi(\xi)-\xi| & =-2(|\xi-a \eta|-b|\eta|), \quad \xi \in M_{a, b}^{\varepsilon,-}(\eta) . \\
\frac{|\phi(\xi)-a \eta|}{|\xi-a \eta|} & \leq 2, \quad \xi \in M_{a, b}^{\varepsilon,-}(\eta) \text { and }|\eta|>1 . \\
\mid J_{\phi^{-1}\left(\xi^{\prime}\right) \mid} & =\frac{1}{\left|J_{\phi}\left(\phi^{-1}\left(\xi^{\prime}\right)\right)\right|} \\
& =\frac{1}{1+2 \frac{b|\eta|-\left|\phi^{-1}\left(\xi^{\prime}\right)-a \eta\right|}{\left|\phi^{-1}\left(\xi^{\prime}\right)-a \eta\right|}} \leq 2 \text { if }|\eta|>1 .
\end{aligned}
$$

Proof of Proposition 3.10. Let $q_{1}, q_{2}$ be as in the hypothesis and let $(f, g)=\left(q_{1}, q_{2}\right)$, $\left(q_{1}, R_{j} R_{k} q_{2}\right)$ or $\left(R_{j} R_{k} q_{1}, q_{2}\right)$.

Using (3.31)-(3.34) we can rewrite

$$
\widehat{\mathcal{V}}(f, g)(\eta)=\lim _{\varepsilon \rightarrow 0, \varepsilon>0}\left\{\widehat{I}_{1}^{\varepsilon}(f, g)(\eta)-2 \widehat{I}_{2}^{\varepsilon}(f, g)(\eta)-2 \widehat{I}_{3}^{\varepsilon}(f, g)(\eta)\right\},
$$

where

$$
\begin{aligned}
& \widehat{I}_{1}^{\varepsilon}(f, g)(\eta)=\int_{M_{a, b}^{\varepsilon,-}(\eta)} \frac{[\widehat{f}(\phi(\xi)) \widehat{g}(\eta-\phi(\xi))-\widehat{f}(\xi) \widehat{g}(\eta-\xi)]}{|\phi(\xi)-a \eta|^{2}-b^{2}|\eta|^{2}}\left|J_{\phi}(\xi)\right| d \xi, \\
& \widehat{I}_{2}^{\varepsilon}(f, g)(\eta)=\int_{M_{a, b}^{\varepsilon,-}(\eta)} \frac{\widehat{f}(\xi) \widehat{g}(\eta-\xi)}{(|\phi(\xi)-a \eta|+b|\eta|)|\xi-a \eta|} d \xi,
\end{aligned}
$$

and

$$
\widehat{I}_{3}^{\varepsilon}(f, g)(\eta)=\int_{M_{a, b}^{\varepsilon,-}(\eta)} \frac{\widehat{f}(\xi) \widehat{g}(\eta-\xi)}{(|\xi-a \eta|+b|\eta|)(|\phi(\xi)-a \eta|+b|\eta|)} d \xi
$$


If $\xi \in M_{a, b}^{\varepsilon}(\eta)$, then

$$
|\xi-a \eta| \geq \frac{b}{2}, \quad \text { when }|\eta|>1
$$

then for $i=2,3$,

$$
\chi_{\{\eta:|\eta|>1\}}(\eta)\left|\widehat{I}_{i}^{\varepsilon}(f, g)(\eta)\right| \leq C(a, b) \widehat{T}_{a, b}^{\varepsilon}(f, g)(\eta),
$$

and by Lemma 3.13,

$\left\|\mathcal{F}^{-1}\left(\chi_{\{\eta:|\eta|>1\}}(\cdot) \widehat{I}_{i}^{\varepsilon}(f, g)(\cdot)\right)\right\|_{W^{\alpha, 2}} \leq C\left\{\|g\|_{L^{p}}\|f\|_{W^{\alpha-1 / l}}+\|f\|_{L^{p}}\|g\|_{W^{\alpha-1 / l, 2}}\right\}$.

For $i=1,2$, since $\left|\widehat{R_{j} R_{k}} q_{i}\right| \leq\left|\widehat{q}_{i}\right|$ and $\left\|q_{i}\right\|_{L^{p}} \leq C\left\|q_{i}\right\|_{L^{2}}, p \leq 2$, we have

$$
\left\|\mathcal{F}^{-1}\left(\chi_{\{\eta:|\eta|>1\}}(\cdot) \widehat{I}_{i}^{\varepsilon}(f, g)(\cdot)\right)\right\|_{W^{\alpha, 2}} \leq C\left\|q_{1}\right\|_{W^{\alpha-1 / l, 2}}\left\|q_{2}\right\|_{W^{\alpha-1 / l}} .
$$

Now we will study $I_{1}^{\varepsilon}(f, g)$. By (3.32),

$$
\begin{aligned}
\widehat{I_{1}^{\varepsilon}}(f, g)(\eta)= & \int_{M_{a, b}^{\varepsilon,-}(\eta)} \frac{\widehat{f}(\phi(\xi)) \widehat{g}(\eta-\phi(\xi))-\widehat{f}(\xi) \widehat{g}(\eta-\xi)}{|\phi(\xi)-a \eta|^{2}-b^{2}|\eta|^{2}} d \xi \\
& -2 \int_{M_{a, b}^{\varepsilon,-}(\eta)} \frac{\widehat{f}(\phi(\xi)) \widehat{g}(\eta-\phi(\xi))-\widehat{f}(\xi) \widehat{g}(\eta-\xi)}{(|\phi(\xi)-a \eta|+b|\eta|)|\xi-a \eta|} d \xi \\
= & \widehat{I}_{1,1}^{\varepsilon}(f, g)(\eta)-2 \widehat{I}_{1,2}^{\varepsilon}(f, g)(\eta)+2 \widehat{I}_{1,3}^{\varepsilon}(f, g)(\eta),
\end{aligned}
$$

where

$$
\begin{aligned}
& \widehat{I}_{1,1}^{\varepsilon}(f, g)(\eta)=\int_{M_{a, b}^{\varepsilon,-}(\eta)} \frac{\widehat{f}(\phi(\xi)) \widehat{g}(\eta-\phi(\xi))-\widehat{f}(\xi) \widehat{g}(\eta-\xi)}{|\phi(\xi)-a \eta|^{2}-b^{2}|\eta|^{2}} d \xi \\
& \widehat{I}_{1,2}^{\varepsilon}(f, g)(\eta)=\int_{M_{a, b}^{\varepsilon,-}(\eta)} \frac{\widehat{f}(\phi(\xi)) \widehat{g}(\eta-\phi(\xi))}{(|\phi(\xi)-a \eta|+b|\eta|)|\xi-a \eta|} d \xi \\
& \widehat{I}_{1,3}^{\varepsilon}(f, g)(\eta)=\int_{M_{a, b}^{\varepsilon,-}(\eta)} \frac{\widehat{f}(\xi) \widehat{g}(\eta-\xi)}{(|\phi(\xi)-a \eta|+b|\eta|)|\xi-a \eta|} d \xi .
\end{aligned}
$$

We have, for $i=2,3$,

$$
\chi_{\{\eta:|\eta|>1\}}(\eta)\left|\widehat{I}_{1, i}^{\varepsilon}(f, g)(\eta)\right| \leq C \widehat{T}_{a, b}^{\varepsilon}(f, g)(\eta) .
$$

Hence,

$$
\left\|\mathcal{F}^{-1}\left(\chi_{\{\eta:|\eta|>1\}}(\cdot) \widehat{I}_{1, i}^{\varepsilon}(f, g)(\cdot)\right)\right\|_{W^{\alpha, 2}} \leq C\left\|q_{1}\right\|_{W^{\alpha-1 / l, 2}}\left\|q_{2}\right\|_{W^{\alpha-1 / l, 2}}, \quad i=2,3 .
$$

Now we treat $\widehat{I}_{1,1}^{\varepsilon}(f, g)(\eta)$ :

$$
\begin{aligned}
\widehat{I}_{1,1}^{\varepsilon}(f, g)(\eta)= & \int_{M_{a, b}^{\varepsilon,-}(\eta)} \frac{\widehat{f}(\phi(\xi))[\widehat{g}(\eta-\phi(\xi))-\widehat{g}(\eta-\xi)]}{|\phi(\xi)-a \eta|^{2}-b^{2}|\eta|^{2}} d \xi \\
& +\int_{M_{a, b}^{\varepsilon,-}(\eta)} \frac{[\widehat{f}(\phi(\xi))-\widehat{f}(\xi)] \widehat{g}(\eta-\xi)}{|\phi(\xi)-a \eta|^{2}-b^{2}|\eta|^{2}} d \xi=\widehat{J}_{1}^{\varepsilon}(f, g)(\eta)+\widehat{J}_{2}^{\varepsilon}(f, g)(\eta) .
\end{aligned}
$$


Consider first $(f, g)=\left(R_{j} R_{k} q_{1}, q_{2}\right)$.

Notice that since $q_{2} \in L^{r}\left(\mathbb{R}^{2}\right)$ with $r>1$ has compact support then $x_{i} q_{2} \in$ $L^{r}\left(\mathbb{R}^{2}\right)$ for $i=1,2$, and we can assume that $r \leq 2$. Then $\nabla \widehat{q}_{i} \in W^{1, p}\left(\mathbb{R}^{2}\right)$ for some $p>1$, and by (3.17), (3.33) and (3.34) we have

$$
\begin{aligned}
& \left|\widehat{J}_{1}^{\varepsilon}\left(R_{j} R_{k} q_{1}, q_{2}\right)(\eta)\right| \leq \int_{M_{a, b}^{\varepsilon,-}(\eta)} \frac{\left|\widehat{q_{1}}(\phi(\xi))\right| M\left(\nabla \widehat{q_{2}}\right)(\eta-\phi(\xi))}{|\phi(\xi)-a \eta|+b|\eta|} d \xi \\
& \quad+\int_{M_{a, b}^{\varepsilon,-}(\eta)} \frac{\left|\widehat{q_{1}}(\phi(\xi))\right| M\left(\nabla \widehat{q_{2}}\right)(\eta-\xi)}{|\phi(\xi)-a \eta|+b|\eta|} d \xi \\
& \leq C\left\{\frac{1}{|\eta|} \int_{M_{a, b}^{\varepsilon,+}(\eta)}\left|\widehat{q_{1}}(\xi)\right| M\left(\nabla \widehat{q_{2}}\right)(\eta-\xi) d \xi+\int_{M_{a, b}^{\varepsilon,-}(\eta)} \frac{\left|\widehat{q_{1}}(\phi(\xi))\right| M\left(\nabla \widehat{q_{2}}\right)(\eta-\xi)}{|\phi(\xi)-a \eta|+b|\eta|} d \xi\right\} \\
& =C\left\{\widehat{J}_{1,1}^{\varepsilon}\left(q_{1}, q_{2}\right)(\eta)+\widehat{J}_{1,2}^{\varepsilon}\left(q_{1}, q_{2}\right)(\eta)\right\} .
\end{aligned}
$$

Since

$$
\left|\chi_{\{|\cdot|>1\}}(\cdot) \widehat{J}_{1,1}^{\varepsilon}\left(q_{1}, q_{2}\right)(\eta)\right| \leq C \widehat{T}_{a, b}^{\varepsilon}\left(q_{1}, \mathcal{F}^{-1} M\left(\nabla \widehat{q_{2}}\right)\right)(\eta),
$$

then by Lemma 3.13 and (3.13), it follows that

$$
\begin{aligned}
& \left\|\mathcal{F}^{-1}\left(\chi_{\{|\cdot|>1\}}(\cdot) \widehat{J}_{1,1}^{\varepsilon}\left(q_{1}, q_{2}\right)(\cdot)\right)\right\|_{W^{\alpha, 2}} \leq C\left\|T_{a, b}^{\varepsilon}\left(q_{1}, \mathcal{F}^{-1} M\left(\nabla \widehat{q_{2}}\right)\right)\right\|_{W^{\alpha, 2}} \\
& \quad \leq C\left\{\left\|q_{1}\right\|_{W^{\alpha-1 / l, 2}}\left\|M\left(\nabla \widehat{q_{2}}\right)\right\|_{L^{2 l}}+\left\|\widehat{q}_{1}\right\|_{L^{2 l}}\left\|\mathcal{F}^{-1}\left(M\left(\nabla \widehat{q_{2}}\right)\right)\right\|_{W^{\alpha-1 / l, 2}}\right\} \\
& \quad \leq C\left\|q_{1}\right\|_{W^{\alpha-1 / l, 2}}\left\|q_{2}\right\|_{W^{\alpha-1 / l, 2}} .
\end{aligned}
$$

On the other hand, since by (3.11) we have $\left|\widehat{q_{1}}(\phi(\xi))\right| \leq C M \widehat{q_{1}}(\xi)$, then

$$
\left|\chi_{\{|\cdot|>1\}}(\eta) \widehat{J}_{1,2}^{\varepsilon}\left(R_{j} R_{k} q_{1}, q_{2}\right)(\eta)\right| \leq C \widehat{T}_{a, b}^{\varepsilon}\left(\mathcal{F}^{-1} M \widehat{q}_{1}, \mathcal{F}^{-1}\left(M\left(\nabla \widehat{q_{2}}\right)\right)(\eta),\right.
$$

and again by Lemma 3.13,

$$
\left\|\mathcal{F}^{-1}\left(\chi_{\{|\cdot|>1\}}(\cdot) \widehat{J}_{1,2}^{\varepsilon}\left(q_{1}, q_{2}\right)(\cdot)\right)\right\|_{W^{\alpha, 2}} \leq C\left\|q_{1}\right\|_{W^{\alpha-1 / l, 2}}\left\|q_{2}\right\|_{W^{\alpha-1 / l, 2}}
$$

Similarly we can prove that

$$
\begin{aligned}
& \left|\chi_{\{|\cdot|>1\}}(\eta) \widehat{J}_{1,1}^{\varepsilon}\left(q_{1}, R_{j} R_{k} q_{2}\right)(\eta)\right| \leq C \widehat{T}_{a, b}^{\varepsilon}\left(\widehat{q_{1}}, \mathcal{F}^{-1}\left(M\left(\nabla \widehat{R_{j} R_{k} q_{2}}\right)\right)\right)(\eta), \\
& \left|\chi_{\{|\cdot|>1\}}(\eta) \widehat{J}_{1,2}^{\varepsilon}\left(q_{1}, R_{j} R_{k} q_{2}\right)(\eta)\right| \leq C \widehat{T}_{a, b}^{\varepsilon}\left(\mathcal{F}^{-1} M \widehat{q}_{1}, \mathcal{F}^{-1}\left(M\left(\nabla \widehat{R_{j} R_{k} q_{2}}\right)\right)\right)(\eta) .
\end{aligned}
$$

From (3.35)-(3.38), and Lemma 3.13, we arrive to

$$
\left\|\mathcal{F}^{-1}\left(\chi_{\{|\cdot|>1\}}(\cdot)\left(J_{1}^{\varepsilon}(f, g)\right)^{\widehat{\gamma}}(\cdot)\right)\right\|_{W^{\alpha, 2}} \leq C\left\|q_{1}\right\|_{W^{\alpha-1 / l, 2}}\left\|q_{2}\right\|_{W^{\alpha-1 / l, 2}},
$$

for $(f, g)=\left(R_{j} R_{k} q_{1}, q_{2}\right),\left(q_{1}, R_{j} R_{k} q_{2}\right)$ and the easier case $\left(q_{1}, q_{2}\right)$. 
Finally, we have to estimate $J_{2}^{\varepsilon}(f, g)$. We see that

$$
\begin{aligned}
\widehat{J_{2}^{\varepsilon}}\left(R_{j} R_{k} q_{1}, q_{2}\right)(\eta)= & \int_{M_{a, b}^{\varepsilon,-}} \frac{\left[\frac{\phi(\xi)_{j} \phi(\xi)_{k}}{|\phi(\xi)|^{2}} \widehat{q_{1}}(\phi(\xi))-\frac{\xi_{j} \xi_{k}}{|\xi|^{2}} \widehat{q_{1}}(\xi)\right] \widehat{q_{2}}(\eta-\xi)}{|\phi(\xi)-a \eta|^{2}-b^{2}|\eta|^{2}} d \xi \\
= & \int_{M_{a, b}^{\varepsilon,-}} \frac{\frac{\phi(\xi)_{j} \phi(\xi)_{k}}{|\phi(\xi)|^{2}}\left[\widehat{q_{1}}(\phi(\xi))-\widehat{q_{1}}(\xi)\right] \widehat{q_{2}}(\eta-\xi)}{|\phi(\xi)-a \eta|^{2}-b^{2}|\eta|^{2}} d \xi \\
& +\int_{M_{a, b}^{\varepsilon,-}} \frac{\left[\frac{\phi(\xi)_{j} \phi(\xi)_{k}}{|\phi(\xi)|^{2}}-\frac{\xi_{j} \xi_{k}}{|\xi|^{2}}\right] \widehat{q_{1}}(\xi) \widehat{q_{2}}(\eta-\xi)}{|\phi(\xi)-a \eta|^{2}-b^{2}|\eta|^{2}} d \xi \\
= & \widehat{J}_{2,1}^{\varepsilon}\left(R_{j} R_{k} q_{1}(\phi(\xi)), q_{2}\right)(\eta)+\widehat{J}_{2,2}^{\varepsilon}\left(R_{j} R_{k} q_{1}(\phi(\xi)), q_{2}\right)(\eta) .
\end{aligned}
$$

Then the analysis is similar to the previous cases. Just notice that since $a \neq b$ and using (3.18), we have

$$
\left|\frac{\phi(\xi)_{j} \phi(\xi)_{k}}{|\phi(\xi)|^{2}}-\frac{\xi_{j} \xi_{k}}{|\xi|^{2}}\right| \leq \frac{C}{|b-a||\eta|}|\phi(\xi)-\xi| .
$$

The terms $J_{2}^{\varepsilon}\left(q_{1}, R_{j} R_{k} q_{2}\right)$ and $J_{2}^{\varepsilon}\left(q_{1}, q_{2}\right)$ can be handled in a similar way so that

$$
\left\|\mathcal{F}^{-1}\left(\chi_{\{|\cdot|>1\}}(\cdot) \widehat{J}_{2}^{\varepsilon}(f, g)(\cdot)\right)\right\|_{W^{\alpha, 2}} \leq C\left\|q_{1}\right\|_{W^{\alpha-1 / l, 2}}\left\|q_{2}\right\|_{W^{\alpha-1 / l, 2}},
$$

and the proof of Proposition 3.10 is complete.

\section{Appendix}

In this section we study the convergence for points $(a, b)$ with $b>0,0<a<1$ of the integral $J(a, b)$, see $(3.9)$.

Proposition 4.1. $J(a, b)<\infty$ for every $(a, b) \in \mathbb{R} \times(0, \infty)$.

For the proof we will need the following lemma.

Lemma 4.2. Let $\gamma_{1}, \gamma_{2} \in \mathbb{R}$ with $\gamma_{1} \neq \gamma_{2}, \beta<1$ and $I$ a bounded interval on $\mathbb{R}$. We have

$$
\int_{I} \frac{d x}{\left|x-\gamma_{1}\right|^{\beta}\left|x-\gamma_{2}\right|^{\beta}} \leq C(\beta)\left(d^{1-2 \beta}+|I| d^{-2 \beta}\right),
$$

where $d=\left|\gamma_{1}-\gamma_{2}\right|$ and $|I|$ is the measure of $I$.

Proof of Proposition 4.1. If $a \neq b$, then

$$
J(a, b)=\iint_{D} \frac{d t d s}{\left(1+t^{2}\right)^{2-l^{\prime}}\left(1+\left(a^{2}-b^{2}\right)^{2} s^{2}\right)^{2-l^{\prime}}|g(s, t)|^{l^{\prime} / l}},
$$

where $D=\left\{(s, t):-1 /(a+b)^{2}<s<0, t>1\right\}$ and $g$ is given by (3.5). 
If $a=b=r \neq 1 / 2$, then

$$
J(r, r) \leq\left(\frac{C}{r^{2}}\right)^{\alpha l^{\prime}} \int_{1}^{\infty} \int_{-1 /\left(4 r^{2}\right)}^{0} \frac{d s d t}{\left(1+t^{2}\right)^{l^{\prime}-2 l^{\prime} / l}|g(s, t)|^{l^{\prime} / l}}
$$

(see (3.10) for the definition of $g$ in this case). Since $J(1-a, b)=J(a, b)$ it is enough to estimate $J(1-b, b), J(a, a)$ and $J(a, b)$ for $(a, b) \in L$, where

$L=\{(a, b) \in(0,1) \times(0, \infty):\{0<b<a$ and $a<1 / 2\} \cup\{0<b \neq a$ and $a>1 / 2\}\}$

Let $(a, b) \in(0,1) \times(0, \infty)$ and write the function $g$ as

$$
\begin{aligned}
g(s, t)= & (1-a)(a-b) t\left[-(a+b)^{3}(a-b) s^{2}+1\right] \\
& +(a+b)|a-b| a s\left[((1-a)-b) t^{2}-(b+(1-a))\right] .
\end{aligned}
$$

1) Case $(a, b) \in L$.

Given $t \in(1, \infty)$ fixed, let

$$
g_{t}(s)=g(s, t)=q_{1}(t) s^{2}+q_{2}(t) s+q_{3}(t),
$$

where

$$
\begin{aligned}
& q_{1}(t)=-(1-a)(a+b)^{3}(a-b)^{2} t, \\
& q_{2}(t)=(a+b)|a-b| a\left[((1-a)-b) t^{2}-(b+(1-a))\right], \\
& q_{3}(t)=(1-a)(a-b) t .
\end{aligned}
$$

If $(a, b) \in L$ and $t \in(1, \infty)$ is fixed, a straightforward argument, shows that the above polynomial $g_{t}$ has two different real roots $\alpha_{1}(t)$ and $\alpha_{2}(t)$.

We have

$$
d(t)=\left|\alpha_{1}(t)-\alpha_{2}(t)\right|=\frac{\sqrt{\Delta(t)}}{\left|q_{1}(t)\right|}=(a+b) \frac{|a-b| \sqrt{f(t)}}{\left|q_{1}(t)\right|},
$$

with

$$
\Delta(t)=(a+b)^{2}(a-b)^{2} f(t), \quad f(t)=r_{1} t^{4}+r_{2} t^{2}+r_{3}>0 \quad \text { if } t \in[1, \infty),
$$

where

$$
\begin{aligned}
& r_{1}=a^{2}((1-a)-b)^{2}, \\
& r_{2}=2\left[2(1-a)^{2}\left(a^{2}-b^{2}\right)-a^{2}\left((1-a)^{2}-b^{2}\right)\right], \\
& r_{3}=a^{2}(b+(1-a))^{2} .
\end{aligned}
$$

If $r_{2} \geq 0$ then $f(t) \geq r_{1} t^{4}$, and hence

$$
d(t) \geq \frac{(a+b)(a-b) a|1-a-b| t^{2}}{(1-a)(a+b)^{3}(a-b)^{2} t}=\frac{a|1-a-b| t}{(1-a)(a+b)^{2}|a-b|}
$$


For $r_{2} \leq 0$ we consider the function

$$
F(t)=\frac{t^{4}+A t^{2}+B}{t^{4}}, \quad t \in[1, \infty), A<0, A^{2}<4 B .
$$

It can be easily seen that if $\sqrt{-2 B / A}<1$ then $F(t) \geq F(1)$ and hence $t^{4}+A t^{2}+$ $B \geq t^{4}(1+A+B)$, while if $\sqrt{-2 B / A}>1$ then $F(t) \geq F(\sqrt{-2 B / A})$ and hence $t^{4}+A t^{2}+B \geq t^{4}\left(\frac{4 B-A^{2}}{4 B}\right)$.

Applying this to our function $f(t)$, we see that if $\sqrt{-2 r_{3} / r_{2}}<1$ then $f(t) \geq$ $\left(4(2 a-1)\left(b^{2}-a^{2}\right)+4 a^{2}\right) t^{4}$ and hence

$$
d(t) \geq \frac{2 \sqrt{(2 a-1)\left(b^{2}-a^{2}\right)+a^{4}} t}{(1-a)(a+b)^{2}|a-b|} .
$$

For $\sqrt{-2 r_{3} / r_{2}}>1$ then $f(t) \geq \frac{-4(1-a)^{2}\left(a^{2}-b^{2}\right) b^{2}(2 a-1)}{a^{2}(b+1-a)^{2}} t^{4}$. We obtain

$$
d(t) \geq \frac{2 b \sqrt{|2 a-1|} t}{a|1-a+b|(a+b)^{3 / 2}|a-b|^{1 / 2}} .
$$

Finally we see that for $(a, b) \in L$ and $t \in[1, \infty)$ we have

$$
\frac{1}{d(t)} \leq\left\{\frac{1}{|a||1-a-b|}+\frac{1}{2 \sqrt{\left|(2 a-1)\left(b^{2}-a^{2}\right)\right|+a^{4}}}+\frac{1}{2 b \sqrt{|2 a-1|}}\right\} \frac{1}{t}=\frac{B(a, b)}{t} .
$$

If we apply Lemma 4.2 and we take $2<l<3$, in order to have $2 l^{\prime} / l-1>0$, we have

$$
\begin{aligned}
J(a, b) & \leq C \int_{1}^{\infty} \frac{1}{t^{4-2 l^{\prime}\left|q_{1}(t)\right|^{l^{\prime} / l}}} \int_{-\frac{1}{(a+b)^{2}}}^{0} \frac{d s}{\left|s-\alpha_{1}(t)\right|^{l^{\prime} / l}\left|s-\alpha_{2}(t)\right|^{l^{\prime} / l}} d y \\
& \leq C\left(l, l^{\prime}\right) \int_{1}^{\infty} \frac{d t}{t^{4-2 l^{\prime}}\left|q_{1}(t)\right|^{l^{\prime} / l} d(t)^{2 l^{\prime} / l-1}}+\frac{C\left(l, l^{\prime}\right)}{(a+b)^{2}} \int_{1}^{\infty} \frac{d t}{t^{4-2 l^{\prime}}\left|q_{1}(t)\right|^{l^{\prime} / l} d(t)^{2 l^{\prime} / l}} .
\end{aligned}
$$

And hence,

$$
J(a, b) \leq\left(\frac{1}{(1-a)(a+b)^{3}|a-b|^{2}}\right)^{l^{\prime} / l}\left(B(a, b)^{l^{\prime} / l-1}+\frac{B(a, b)^{l^{\prime} / l}}{(a+b)^{2}}\right) .
$$

2) Case $a=1-b$.

For $1 / 2<b<1$,

$$
g^{s}(t)=g(s, t)=b(1-2 b)\left(\left(1+(2 b-1) s^{2}\right) t+2(1-b) s\right) .
$$

Since $-1 \leq s \leq 0$ and $t>1$ we have that $\left(1+(2 b-1) s^{2}\right) t+2(1-b) s \geq 2 b-1$. Hence $\left|g^{s}(t)\right| \geq b(2 b-1)^{2}$ and

$$
J(1-b, b) \leq \frac{C}{\left(b(2 b-1)^{2}\right)^{l^{\prime} / l}} .
$$


For $0<b<1 / 2$,

$$
g^{s}(t)=b(1-2 b)\left(\left(1+(2 b-1) s^{2}\right) t-2(1-b) s\right) .
$$

Since $1+(2 b-1) s^{2} \geq 0$ then $\left|g^{s}(t)\right| \geq b|1-2 b||1-b||s|$, and so

$$
J(1-b, b) \leq \frac{C}{(b|1-2 b||1-b|)^{l^{\prime} / l}} .
$$

3) Case $a=b=r \neq 1 / 2$.

For $1 / 2<r<1$, letting $u=(1-r) t+2 r^{2} s\left[(1-r) t^{2}-1\right]$ we get

$$
\begin{aligned}
J(r, r) & \leq\left(\frac{C}{r^{2}}\right)^{\alpha l^{\prime}+1} \int_{1}^{\infty} \int_{(1-r) t-1 / 2\left[\left(1-2 r_{t}^{2}-1\right]\right.}^{(1-r) t} \frac{d u}{|u|^{l^{\prime} / l}} \frac{d t}{\left[(1-2 r) t^{2}-1\right]\left(1+t^{2}\right)^{l^{\prime}(1-2 / l)}} \\
& \leq\left(\frac{C}{r^{2}}\right)^{\alpha l^{\prime}+1} \frac{|1-2 r|^{1-l^{\prime} / l}}{|1-2 r|} \int_{1}^{\infty} \frac{d t}{t^{2}} \leq\left(\frac{C}{r^{2}}\right)^{\alpha l^{\prime}+1}|1-2 r|^{-l^{\prime} / l} .
\end{aligned}
$$

For $0<r<1 / 2$ we have

$$
J(r, r) \leq\left(\frac{C}{r^{2}}\right)^{\alpha l^{\prime}} \int_{1}^{\infty} \int_{\frac{-1}{4 r^{2}}}^{0} \frac{d s d t}{\left(1+t^{2}\right)^{l^{\prime}-2 l^{\prime} / l}\left|(1-r) t+2 r^{2} s\left[(1-r) t^{2}-1\right]\right|^{l^{\prime} / l}} .
$$

Let

$\mathrm{I}=\int_{\{t>1\} \cap\left\{\left|(1-2 r) t^{2}-1\right|<1-r\right\}} \int_{\frac{-1}{4 r^{2}}}^{0} \frac{d s d t}{\left(1+t^{2}\right)^{l^{\prime}-2 l^{\prime} / l}\left|(1-r) t+2 r^{2} s\left[(1-r) t^{2}-1\right]\right|^{l^{\prime} / l}}$

and

$\mathrm{II}=\int_{\{t>1\} \cap\left\{\left|(1-2 r) t^{2}-1\right|>1-r\right\}} \int_{\frac{-1}{4 r^{2}}}^{0} \frac{d s d t}{\left(1+t^{2}\right)^{l^{\prime}-2 l^{\prime} / l}\left|(1-r) t+2 r^{2} s\left[(1-r) t^{2}-1\right]\right|^{l^{\prime} / l}}$.

To estimate I, notice that if $t \in\{t>1\} \cap\left\{\left|(1-2 r) t^{2}-1\right|<1-r\right\}$ then

$\left|(1-r) t+2 r^{2} s\left[(1-r) t^{2}-1\right]\right|>(1-r)\left(t-\frac{1}{2}\right)$ and $\left(\frac{r}{1-2 r}\right)^{1 / 2}<t<\left(\frac{2-r}{1-2 r}\right)^{1 / 2}$.

Hence,

$$
I \leq \int_{\left(\frac{r}{1-2 r}\right)^{1 / 2}}^{\left(\frac{2-r}{1-2 r}\right)^{1 / 2}} \int_{\frac{-1}{4 r^{2}}}^{0} \frac{d s d t}{\left((1-r)\left(t-\frac{1}{2}\right)\right)^{l^{\prime} / l} t^{2 l^{\prime}(1-2 / l)}} \leq C \frac{(1-r)^{-l^{\prime} / l}}{(1-2 r)^{\frac{1}{2}\left(-l^{\prime}(2-3 / l)+1\right) r^{2}}} .
$$

To estimate II we make the change of variable $u=(1-r) t+2 r^{2} s\left((1-2 r) t^{2}-1\right)$.

Notice that for $t \in\{t>1\} \cap\left\{\left|(1-2 r) t^{2}-1\right|>1-r\right\}, t^{2} \neq 1-2 r$.

$$
\begin{aligned}
\mathrm{II}= & \int_{1}^{\left(\frac{r}{1-2 r}\right)^{1 / 2}} \int_{(1-r) t}^{(1-r) t-\frac{1}{2}\left((1-2 r) t^{2}-1\right)} \frac{d u d t}{u^{l^{\prime} / l} 2 r^{2}\left[1-(1-2 r) t^{2}\right] t^{2 l^{\prime}(1-2 / l)}} \\
& +\int_{\left(\frac{2-r}{1-2 r}\right)^{1 / 2}}^{\infty} \int_{(1-r) t-\frac{1}{2}\left((1-2 r) t^{2}-1\right)}^{(1-r) t} \frac{d u d t}{u^{l^{\prime} / l} 2 r^{2}\left[1-(1-2 r) t^{2}\right] t^{2 l^{\prime}(1-2 / l)}} \\
\leq & C \frac{(1-2 r)^{-l^{\prime} / l}}{r^{2}}
\end{aligned}
$$


Hence we see that

$$
J(r, r) \leq\left(\frac{C}{r^{2}}\right)^{\alpha l^{\prime}+1}|1-2 r|^{-l^{\prime} / l} .
$$

Remark 4.3. Direct calculations show that for any fixed $a \in \mathbb{R}$, the function $A_{l}(a, r)$ is integrable in any finite interval in $(0, \infty)$ not containing $r=-a$.

\section{References}

[1] Barceló, J. A., Folch-Gabayet, M., Pérez-Esteva, S., Ruiz, A. And VileLA, M. C.: A Born approximation for live load in Navier elasticity. SIAM J. Math. Anal. 44 (2012), no. 4, 2824-2846.

[2] Barceló, J. A., Folch-Gabayet, M., Pérez-Esteva, S., Ruiz, A. And VileLA, M. C.: Limiting absorption principles for the Navier equation in elasticity. Ann. Sc. Norm. Super. Pisa Cl. Sci. (5) 11 (2012), no. 4, 817-842.

[3] Grisvard, P.: Elliptic problems in nonsmooth domains. Monographs and Studies in Mathematics 24, Pitman, Boston, MA, 1985.

[4] Hajlasz, P.: Sobolev space on an arbitrary metric space. Potential Anal. 5 (1996), no. 4, 403-415.

[5] Hartman, P., And Wilcox, C.: On solutions of the Helmhotz equation in exterior domains. Math. Z. 75 (1960/1961), 228-255.

[6] Kupradze, V.,D., Gegelia, T. G., Basheleı̌shvili, M. O. And BurchuLADZE, T. V.: Three-dimensional problems of the mathematical theory of elasticity and thermoelasticity. North-Holland Series in Applied Mathematical and Mechanics 25, North-Holland Publishing, Amsterdam-New York, 1979.

[7] Mochizuki, K.: Eigenfunction expansions associated with the Schrödinger operator with a complex potential and the scattering theory. Publ. Res. Inst. Math. Sci. Ser. A $4(1968 / 69), 419-466$.

[8] Ola P., PÄIvÄrinta, L. ANd V. Serov, V.: Recovering singularities from backscattering in two dimensions. Comm. Partial Differential Equations 26 (2001), no. 3-4, 697-715.

[9] PÄIvÄrinta, L. AND SERov, V.: Recovery of singularities of a multidimensional scattering potential. SIAM J. Math. Anal. 29 (1998), no. 3, 697-711.

[10] PÄrvärinta, L. And Somersalo, E.: Inversion of discontinuities for the Schrödinger equation in three dimensions. SIAM J. Math. Anal. 22 (1991), no. 2, 480-499.

[11] Reyes, J. M.: Problema inverso de scattering para la ecuación de Schrödinger: reconstrucción parcial del potencial a partir de datos de retrodispersión en $2 D$ y $3 D$. Ph.D. Thesis, Universidad Autónoma de Madrid, Spain, 2007.

[12] Reyes, J. M.: Inverse backscattering for the Schrödinger equation in 2D. Inverse Problems 23 (2007), no. 2, 625-643.

[13] Reyes, J. M. And Ruiz, A.: Reconstruction of the singurarities of a potential from backscattering data in 2D and 3D. Inverse Probl. Imaging 6 (2012), no. 2, 321-355.

[14] Ruiz, A.: Harmonic analysis and inverse problems (Summer School of Oulu, 2002). http://www.uam.es/gruposinv/inversos/publicaciones/Inverseproblems.pdf.

[15] Ruiz, A. And Vargas A.: Partial recovery of a potential from backscattering data. Comm. Partial Differential Equations 30 (2005), no. 1-3, 67-96. 
[16] Tomas, P. A.: A restriction theorem for the Fourier transform. Bull. Amer. Math. Soc. 81 (1975), 477-478.

[17] Stein, E. M.: Harmonic analysis: real-variable methods, orthogonality, and oscillatory integrals. Princeton Mathematical Series 43, Monographs in Harmonic Analysis III, Princeton University Press, Princeton, NJ, 1993.

Received January 29, 2014.

Juan Antonio Barceló: ETSI de Caminos, Universidad Politécnica de Madrid, 28040 Madrid, Spain.

E-mail: juanantonio.barcelo@upm.es

Magali Folch-Gabayet: Instituto de Matemáticas, Universidad Nacional Autónoma de México, Ciudad Universitaria, México D.F., 04510, México.

E-mail: folchgab@matem.unam.mx

Salvador Pérez-Esteva: Instituto de Matemáticas, Unidad Cuernavaca, Universidad Nacional Autónoma de México, A.P. 273-3 ADMON 3, Cuernavaca, Mor., 62251, México.

E-mail: salvador@im.unam.mx

Alberto Ruiz: Departamento de Matemáticas, Universidad Autónoma de Madrid, 28049, Madrid, Spain.

E-mail: alberto.ruiz@uam.es

Mari Cruz Vilela: Departamento de Matemática Aplicada, Universidad de Valladolid, Plaza Santa Eulalia 9 y 11, 40005 Segovia, Spain.

E-mail: maricruz@eii.uva.es

Partially supported by the mexican grant PAPIIT-UNAM IN100512. The first, fourth and fifth authors were supported by Spanish Grant MTM2011-28198. 